\title{
EXPECTATIVAS RACIONAIS EM MODELOS MACROECONOMÉTRICOS PARA A ECONOMIA BRASILEIRA *
}

\author{
P. Guedes e J. L. Mascolo **
}

\section{1 - Introdução}

Elabora-se, neste trabalho, uma seqüência de modelos macroecométricos de pequeno porte, integrando duas importantes linhas de pesquisa: (i) os modelos macroeconômicos de Lucas-Sargent para análise de flutuações cíclicas, com base na hipótese de expectativas racionais; ( ii ) a abordagem de Zellner e Palm para sistemas dinâmicos de equações simultâneas, combinando a formulação tradicional de equações estruturais com análise de séries temporais.

Impondo restrições a um processo multivariado de séries temporais com base em teoria econômica, isto é, considerando algumas das variáveis do vetor estocástico como endógenas e as demais como puramente exógenas, e admitindo distintos processos de formação de expectativas, obtêm-se as formas estruturais alternativas do sistema, bem como as funções de transferência e equações finais associadas às mesmas.

As implicações testáveis de cada forma estrutural são as ordens das funções de transferência e equações finais para as variáveis endógenas, isto é, produto real, taxas de juros e taxa de inflação. É possivel, portanto, identificar os modelos cujas implicações são compativeis com as informações contidas nos dados e, conseqüentemente, as hipóteses econômicas que não são rejeitadas pela evidência empírica no Brasil.

\section{2 - Metodologias para formulação $\theta$ análise de sistemas dinâmicos de equações simultâneas}

Em seu artigo clássico (1974), Zellner e Palm apresentam uma nova abordagem para o estudo de modelos dinâmicos, baseada em análise de séries temporais e na formulação tradicional de equações estruturais.

* Os autores agradecem a Ricardo Coqueiro e Antonio Perrota por sua assistência computacional.

* Da Fundação Centro de Estudos do Comércio Exterior (CECEX) e Pontifícia Universidade Católica do Rio de Janeiro (PUC-RJ). 
Segundo Quenouille (1957), um conjunto de variáveis $z_{1 t^{\prime}} z_{2 t}, \ldots$, $z_{n t}$ gerado por um processo linear multivariado de séries temporais, pode ser representado por um modelo MARMA (multivariate autoregressive moving average) do tipo:

$$
[H(L)] \vec{z}_{t}=[F(L)] \vec{\epsilon}_{t} \text {, }
$$

onde $t=1,2, \ldots, T ; \vec{z}_{t}=\left(z_{1}, z_{2 t}, \ldots, z_{n t}\right)$ é um vetor de variáveis estocásticas; $\vec{\epsilon}_{\mathrm{t}}=\left(\epsilon_{1 \mathrm{t}}, \epsilon_{2 \mathrm{t}}, \ldots, \epsilon_{\mathrm{nt}}\right)$ é um vetor de erros aleatórios com média zero, não correlacionados serialmente e cuja matriz de variância-covariância é dada pela matriz identidade, isto é, $E \vec{\epsilon}_{\mathrm{t}}=\overrightarrow{0}$ e $E \vec{\epsilon}_{\mathrm{r}} \vec{\epsilon}_{\mathrm{s}}^{\prime}{ }_{\mathrm{s}}=\delta_{\mathrm{rs}} 1$, onde $\mathrm{r}$ e $\mathrm{s} \in(1,2, \ldots$ $\ldots, n), ~ I$ é a matriz identidade e $\delta_{r s}^{r}$ é o delta de Kronecker. [ $\left.H(L)\right]$ e $[F(L)]$ são matrizes de ordem $n \times n$, de posto máximo, cujos elementos são polinômios no operador de defasagens $L$.

Ainda de acordo com Quenouille, as equações em (2.1) podem ser resolvidas para $\vec{z}_{t^{\prime}}$ com as variáveis individuais seguindo processos ARMA univariados consistentes com o processo con junto descrito em ( 2.1 ):

$$
|H(L)| \vec{z}_{t}=[H(L)]^{*}[F(L)] \vec{\epsilon}_{t^{\prime}}
$$

onde $|H(L)|$ é o determinante da matriz não singular $\left[H(L)\right.$ ] e $[H(L)]^{*}$ é a matriz adjunta associada à mesma.

Segundo Zellner e Palm, o processo multivariado linear de séries temporais pode ser transformado num sistema dinâmico de equações simultâneas, desde que restrições baseadas em teoria econômica se jam impostas a $[H(L)]$ e $[F(L)]$ em $(2.1)$. Desta forma, as variáveis $\vec{y}_{t}$ em $\vec{z}_{t}$ podem ser vistas como endógenas, ao passo que as demais variáveis $\vec{x}_{t}$ serão vistas como puramente exógenas, isto é, seguem um processo estocástico independente. Neste caso, pode ser adotada a seguinte partição para (2.1):

$$
\left(\begin{array}{l}
{\left[H_{11}(L)\right]\left[H_{12}(L)\right]} \\
\left.\left[H_{21}(L)\right]\left[H_{22}(L)\right]\right)
\end{array}\right)\left[\begin{array}{l}
\vec{y}_{t} \\
\vec{x}_{t}
\end{array}\right]=\left(\begin{array}{c}
{\left[F_{11}(L)\right]\left[F_{12}(L)\right]} \\
{\left[F_{21}(L)\right]\left[F_{22}(L)\right]}
\end{array}\right)\left[\begin{array}{c}
\vec{u}_{t} \\
\overrightarrow{l_{t}}
\end{array}\right]
$$

onde $\left[H_{11}(L)\right],\left[H_{22}(L)\right],\left[F_{11}(L)\right]$ e $\left[F_{22}(L)\right]$ são matrizes quadradas e as demais possuem as dimensões apropriadas. $A$ hipótese de que o vetor $\vec{y}_{t^{\prime}}$ de ordem $p \times 1$, é endógeno e de que o vetor $\vec{x}_{t}$, de ordem $k \times 1$, é exógeno $(p+k=n)$ faz com que sejam nulas as submatrizes $\left[H_{21}(L)\right],\left[F_{21}(L)\right]$ e $\left[F_{12}(L)\right]$. 
A forma estrutural do sistema dinâmico de equações simultâneas acima é dada por:

$$
\left[H_{11}(L)\right] \vec{y}_{t}+\left[H_{12}(L)\right] \vec{x}_{t}=\left[F_{11}(L)\right] \vec{u}_{t}
$$

e o processo MARMA seguido pelas variáveis exógenas é:

$$
\text { [ } \mathrm{H}_{22}
$$

As equações da forma reduzida do sistema são derivadas das equações estruturais em ( 2.4$)$ :

$$
\begin{aligned}
\vec{y}_{t}= & -\left[\begin{array}{ll}
H^{\circ} & (1)
\end{array}\right]^{-1}\left[\begin{array}{lll}
H^{h} & (
\end{array}\right] \vec{v}_{t}-\left[\begin{array} { l l } 
{ H ^ { \circ } } & { - 1 }
\end{array} \left[\begin{array}{lll}
H & 1
\end{array}\right.\right. \\
& +[H
\end{aligned}
$$

onde [H

zero dos polinômios finitos no operador de defasagens e [H cu jos elementos são as partes homogêneas destes polinômios, de tal forma què

As equações da forma final, no sentido de Theil e Boot (1962), são também derivadas da forma estrutural:

$$
\vec{y}_{t}=-\left[H_{11}(L)\right]^{-1}\left[H_{12}(L)\right] \vec{x}_{t}+\left[H_{11}(L)\right]^{-1}\left[F_{11}(L)\right] \vec{u}_{t}
$$

As funções de transferência (Box e Jenkins, 1970), associadas às equações estruturais do sistema são dadas por:

$$
i H_{11}(L) \mid \vec{y}_{t}=-\left[H_{11}(L)\right] *\left[H_{12}(L)\right] \vec{x}_{t}+\left[H_{11}(L)\right] *\left[F_{11}(L)\right] \vec{u}_{t}
$$

Para se chegar às "equações finais" (Tinbergen, 1940), isto é, modelos ARMA lineares univariados para as variáveis endógenas, o processo MARMA em ( 2.5 ) deve ser substituído em ( 2.7 ):

$$
\begin{aligned}
\left|H_{22}\right| \mid \vec{y}_{t}= & -[H \\
& +\mid H_{22}
\end{aligned}
$$




\section{3 - OS MODELOS MACROECONOMÉTRICOS}

São apresentados nesta Seção os modelos macroeconométricos, essencialmente monetários, cujas implicações serão testadas empiricamente. Admitindo-se de início expectativas adaptativas, duas versões são elaboradas, as quais diferem pela inclusão, ou não, de um termo de persistência para o hiato do produto na equação de oferta agregada.

A inclusão deste termo pode ser justificada, devido à possibilidade de que a oferta responda também a erros passados de previsão, isto é, uma vez fora de sua posição de equilíbrio, a economia não se ajusta instantaneamente. Em seguida, admitindo-se expectativas racionais, deriva-se um modelo simplificado, que é generalizado, posteriormente, através da inclusão de diversos polinômios no operador de defasagens nas equações estruturais.

Os modelos possuem uma estrutura neoclássica comum: equações para oferta e demanda por moeda, uma condição de equilíbrio no mercado monetário, uma equação de Fisher para a determinação da taxa de juros nominal, uma oferta agregada tipo Lucas-Sargent e uma equação para descrever o processo de formação de expectativas.

\section{1 - Um modelo com expectativas adaptativas}

A estrutura geral acima descrita, conjugaaa com a hipótese de expectativas adaptativas e sem um termo de persistência na equação de oferta agregada, tem a seguinte representação:

$$
y_{t}-y_{t}^{n}=\gamma\left(p_{t}-p_{t}^{*}\right)+u_{1 i}
$$

onde

$y_{t}=\ell n \quad$ do produto real

$y_{t}^{n}=\ell n \quad$ do produto potencial

$\boldsymbol{\gamma}$ = parâmetro que mede o desvio entre as taxas de crescimento - observada $\mathrm{e}$ potencial - do produto real como resposta a um ponto percentual de inflação não-antecipada

$p_{t}=\ell$ donivel de preços

$p_{t}^{*}=\ell n \quad$ antecipado do nivel de precos

$u_{1 t}=$ perturbação aleatória (choque real) 
que é a equação de oferta agregada tipo Lucas-Sargent, na qual a inflação não-antecipada explica os desvios entre o produto real e sua tendência natural.

$$
m_{t}^{d}=p_{t}+\alpha_{1} y_{t}-\alpha_{2} i_{t}+u_{2 t}
$$

onde

$m_{t}^{d}=\ell_{n} \quad$ da demanda nominal por moeda

$\alpha_{1}=$ elasticidade renda da demanda por moeda; $\alpha_{1}>0$

$i_{t} \quad=$ taxa de juros nominal

$\alpha_{2} i_{t}=$ elasticidade juros da demanda por moeda; $\alpha_{2}>0$

$\mathrm{u}_{2 \mathrm{t}}=$ erro aleatório,

que especifica a demanda por encaixes reais como função da renda real e da taxa nominal de juros.

$$
i_{t}=\rho+\left(p_{t}^{*}-p_{t-1}\right)+u_{3 t}
$$

onde

$\rho=$ taxa de juros real

$p_{t}^{*}-p_{t-1}=$ taxa

$u_{3 t}=$ erro aleatório,

que é a equação de Fişher. Trata-se de uma representação da teoria neoclássica de determinação da taxa de juros nominal, segundo a qual o componente real é determinado por fatores reais (poupança e investimento) ao passo que o prêmio inflacionário é determinado pela taxa de inflação esperada (fatores monetários).

$$
m_{t}^{s}=h_{t}+\alpha_{3} i_{t}+u_{4 t^{\prime}}
$$

onde

$m_{t}^{s}=\ell n \quad$ da oferta nominal de moeda

$h_{t}=\ell n \quad$ da base monetária 
$\alpha_{3}=$ parâme tro que mede o desvio entre as taxas de crescimento, da oferta de moeda e da base mone tária, devido a um aumen to de um pon to percentual na taxa nominal de juros,

que descreve o proœsso de oferta de moeda como função da base monetária e do multip licador monetário.

$$
p_{t}^{*}=p_{t-1}^{*}+\beta\left(p_{t-1}-p_{t-1}^{*}\right)
$$

onde

$\beta=$ coeficiente de ajustamento das expectativas; $0<\beta \leqslant 1$,

que descreve o mecanismo de expec tativas adaptativas.

$$
m_{t}^{d}=m_{t}^{s}
$$

que es tabelece a condição de equilíbrio no mercado monetário.

Deve-se eliminar a variável não observável da forma estrutural. De acordo com ( 6 ), pode-se igualar (2) e (4) obtendo-se uma demanda agregada no mercado de bens e serviços, cujos parâmetros são $i_{t}$ e $h_{t}$. Diferenciando a equação obtida, elimina-se $m_{t}^{s}$ e $m_{t}^{d}$, chegandose à primeira equação da nova forma es trutural:

$$
r p_{t}+\alpha_{1} r y_{t}=\left(\alpha_{2}+\alpha_{3}\right) \Delta i_{t}+r h_{t}-(1-L)\left(u_{2 t}-u_{4 t}\right)
$$

onde

$L \quad \equiv$ operador de defasagens

$\Delta \equiv(1-\mathrm{L})$

$r p_{t} \equiv \Delta p_{t}=$ taxa de inflação

$r y_{t} \equiv \Delta y_{t}=$ taxa de crescimen to d o produto real

$r h_{t_{1}} \equiv \Delta h_{t}=$ taxa de crescimento da base monetária

Resta, ainda, eliminar $p_{t}^{*}$ em (1) e (3). Diferenciando (3) e multiplicando a equação resultante por $[1-(1-\beta) L]$, tem-se: 


$$
\begin{aligned}
{\left[1-(1-\beta) L j(1-L) i_{t}=\right.} & {[1-(1-\beta) L](1-L) L p_{t}^{*}-(1-L)[1-(1-\beta) L] L p_{t}+} \\
& +(1-L)[1-(1-\beta) L] u_{3 t}
\end{aligned}
$$

Rearrumando os termos em (5) e multiplicando por (1-L), tem-se:

$$
\left[1-(1-\beta) L j(1-L) p_{t}^{*}=(1-L) \beta L p_{t}\right.
$$

Levando ( 9 ) em ( 8 ), elimina-se a variável não-observável $p_{t}^{*}$ na equação da taxa de juros, obtendo-se a segunda equação da nova forma estrutural:

$$
\left[1-(1-\beta) L j \Delta i_{t}=-(1-\beta)(1-L) L r p_{t}+(1-L)[1-(1-\beta) L] u_{3 t}\right.
$$

Multiplicando (1) por $[1-(1-\beta) L](1-L)$ e usando (9), fecha-se o sistema:

$$
\left[1-(1-\beta) L_{j}\left(r y_{t}-r y^{n}\right)=\gamma(1-L) r p_{t}+(1-L)\left[1-(1-\beta) L j u_{1 t}\right.\right.
$$

onde $\begin{array}{ll}r y^{n}=\Delta y_{t}^{n}= & \begin{array}{l}\text { taxa de crescimento do produto potencial (taxa natural de cresci- } \\ \text { mento), suposta constante. }\end{array}\end{array}$ A nova forma estrutural do modelo é dada pelas equações (7), (10) e (11). Em notação matricial tem-se:

$$
\begin{aligned}
& {\left[\begin{array}{ccc}
\alpha_{1} & 1 & -\left(\alpha_{2}+\alpha_{3}\right) \\
{[1-(1-\beta) L]} & -\gamma(1-L) & 0 \\
0 & (1-\beta)(1-L) L & {[1-(1-\beta) L]}
\end{array}\right]\left(\begin{array}{c}
r y_{t} \\
r p_{t} \\
\Delta i_{t}
\end{array}\right)=} \\
& =\left(\begin{array}{c}
0 \\
{[1-(1-\beta) L i} \\
0
\end{array}\right) r y^{n}+\left(\begin{array}{l}
1 \\
0 \\
0
\end{array}\right) m_{t}+\left(\begin{array}{c}
v_{1 t} \\
v_{2 t} \\
v_{3 t}
\end{array}\right)
\end{aligned}
$$

onde 


$$
\begin{aligned}
& v_{1 t}=(1-L)\left(u_{4 t}-u_{2 t}\right) \\
& v_{2 t}=(1-L)\left(1-(1-\beta) L ; u_{1 t}\right. \\
& v_{3 t}=(1-L)\left(1-(1-\beta) L ; u_{3 t}\right.
\end{aligned}
$$

Resolvendo ( 12 ) para $r y_{t}, r p_{t}$ e $\Delta i_{t}$ chega-se às funçōes de transferência associadas ao modelo:

$$
\begin{aligned}
& \left\{\alpha _ { 1 } \gamma ( 1 - L ) \left(1-(1-\beta) L j-\left(\alpha_{2}+\alpha_{3}\right)(1-L)(1-\beta) L\left(1-(1-\beta) L_{j}-\right.\right.\right. \\
& -\left[1-(1-\beta) L:{ }^{2}\right\} r y_{t}=\{-\gamma(1-L)[1-(1-\beta) L]\} r h_{t}-\beta^{2} r y^{n}+ \\
& +\left\{-\gamma(1-L)\left[1 \ldots(1-\beta) L_{i}\right\} v_{1 t}+\left\{-\left[1-(1-\beta) L i-\left(\alpha_{2}+\alpha_{3}\right)(1-\beta)(1-L) L\right\} v_{2 t}+\right.\right. \\
& +\{-\gamma \\
& \left\{-\alpha_{1} \gamma(1-L)-\left(\alpha_{2}+\alpha_{3}\right)(1-L)(1-\beta) L-[1-(1-\beta) L]\right\} r p_{t}= \\
& =\left\{-[1-(1-\beta) L .\} r h_{t}+\alpha_{1} \beta r y^{n}+\left\{-\left[1-(1-\beta) L_{j}\right\} v_{1 t}+\right.\right. \\
& +\alpha_{1} v_{2 t}-\left(\alpha_{2}+\alpha_{3}\right) v_{3 t} \\
& \left\{-\alpha_{1} \gamma(1-L)\left[1-(1-\beta) L,-\left(\alpha_{2}+\alpha_{3}\right)(1-L)(1-\beta) L[1-(1-\beta) L j-\right.\right. \\
& -\left[1-(1-\beta) L,{ }^{2}\right\} \Delta i_{t}=\left\{(1-\beta)(1-L)[1-(1-\beta) L ; L\} r h_{t}+\right. \\
& +\left\{(1-\beta)(1-L)[1-(1-\beta) L I L\} v_{1 t}+\left\{-\alpha_{1}(1-\beta)(1-L) L\right\} v_{2 t}+\right. \\
& +\left\{-\alpha_{1} \gamma(1-L)-[1-(1-\beta) L i\} v_{3 t}\right.
\end{aligned}
$$

Assim sendo, as ordens dos polinómios no operador de defasagens das funçōes de transferência, que são implicações testáveis da forma estrutural em (12), são:

\begin{tabular}{cccc}
\hline Variável & AR & MA para rh & MA para o erro \\
\hline$r y_{t}$ & 3 & 2 & 4 \\
$r p_{t}$ & 2 & 1 & 2 \\
$\Delta i_{t}$ & 3 & 3. & 4 \\
\hline
\end{tabular}


Fazendo a hipótese de que a variável exógena segue um processo ARMA independente dado por $\psi^{\prime} \mathrm{p}(\mathrm{L}) \mathrm{rh}_{\mathrm{t}}=\theta_{\mathrm{q}}(\mathrm{L}) \mathrm{u}_{5 \mathrm{t}}$ e multiplicando as equações $(13),(14)$ e $(15)$ por sp $(L)$, chega-se às equações finais associadas a $(12)$ :

$$
\begin{aligned}
& \psi_{p}(L)\left\{-\alpha_{1} \gamma(1-L)[1-(1-\beta) L]-\left(\alpha_{2}+\alpha_{3}\right)(1-L)(1-\beta) L[1-(1-\beta) L j-\right. \\
& -\left[1-(1-\beta) L_{j}^{2}\right\} r y_{t}=\{-\gamma(1-L)[1-(1-\beta) L]\} \theta_{q}(L) u_{5 t}- \\
& -\phi_{p}(1) \beta^{2} r y^{n}+\left\{-\gamma(1-L)[1-(1-\beta) L\} \psi_{p}(L) v_{1 t}+\right. \\
& +\left\{-\left[1-(1-\beta) L_{j}-\left(\alpha_{2}+\alpha_{3}\right)(1-\beta)(1-L) L\right\} \psi_{p}(L) v_{2 t}+\right. \\
& +\left\{-\gamma(1-L)\left(\alpha_{2}+\alpha_{3}\right)\right\} \psi_{p}(L) v_{3 t} \\
& \phi_{p}(L)\left\{-\alpha_{1} \gamma(1-L)-\left(\alpha_{2}+\alpha_{3}\right)(1-L)(1-\beta) L-\left[1-(1-\beta) L_{j}\right\} r p_{t}=\right. \\
& \cdot\left\{-[1-(1-\beta) L j\} \theta q(L) u_{5 t}+\psi_{p}(1) \alpha_{1} \beta r y^{n}\right. \\
& +\left\{-\left[1-(1-\beta) L_{j}\right\} \cdot r_{p}(L) v_{1 t}+\alpha_{1} \phi_{p}(L) v_{2 t}-\left(\alpha_{2}+\alpha_{3}\right) \psi_{p}(L) v_{3 t} \quad(17)\right. \\
& { }_{p}(L)\left\{-\alpha_{1} \gamma(1-L)[1-(1-\beta) L]-\left(\alpha_{2}+\alpha_{3}\right)(1-L)(1-\beta) L[1-(1-\beta) L]-\right. \\
& -\left[1-(1-\beta) L j{ }^{2}\right\} \Delta_{t}=\left\{(1-\beta)(1-L)\left(1-(1-\beta) L_{1} L\right\} \theta_{q}(L) u_{5 t}+\right. \\
& +\{(1-\beta)(1-L)[1-(1-\beta) L] L\} \phi_{p}(L) v_{1 t}+\left\{-\alpha_{1}(1-\beta)(1-L) L\right\} \psi_{p}(L) v_{2 t}+ \\
& +\left\{-\alpha_{1} \gamma(1-L)-[1-(1-\beta) L]\right\} \phi_{p}(L) v_{3 t}
\end{aligned}
$$

As ordens dos polinômios, por inspeção para as partes $A R$ e aplicando-se o lema de Granger às partes MA, são:

\begin{tabular}{lcc}
\hline Variável & $A R$ & $M A$ \\
\hline$r y_{t}$ & $p+3$ & $\max (q+2 ; p+4)$ \\
$r p_{t}$ & $p+2$ & $\max (q+1 ; p+2)$ \\
$\Delta i_{t}$ & $p+3$ & $\max (q+3 ; p+4)$ \\
$r h_{t}$ & $p$ & $q$ \\
\hline
\end{tabular}


3.2. Um modelo com expectativas adaptativas e persistência no produto real

Desvios entre o produto observado e seu nivel potencial podem não ser totalmente explicados pela inflação não-antecipada no periodo. Erros passados de previsão podem ter deslocado a economia de sua trajetória natural de crescimen. to e, como o ajustamento não se dá de forma instantânea, estes erros podem ser responsáveis por desvios correntes.

Para levar este efeito em consideração, foi introduzido um termo de persistência do hiato

$$
y_{t}-y_{t}^{n}=\gamma\left(p_{t}-p_{t}^{*}\right)+k\left(y_{t-1}-y_{t-1}^{n}\right)+u_{t t}
$$

onde $k \equiv$ coeficiente de persistência do hiato do produto real; $0<k<1$.

O sistema

terior.

A demanda agregada no mercado de bens e serviços e a equação da taxas de juros são obtidas exatamente como na seção anterior (vide equaçōes ( 7 ) e (10)).

Expressando $p_{t}^{*}$ como função de $r p_{t}$ em $(5)$, multiplicando a equação resultante por $\gamma$ e eliminando $p_{t}^{*}$ na equação $(19)$ após multiplicá-la por $[1-(1-\beta) L$, obtém-se uma oferta agregada em função apenas das variáveis observáveis:

$$
\begin{array}{r}
(1-k L)\left[1--(1-\beta) L j r y_{t}-(1-k)\left(1-(1-\beta) L j r y^{n}=\right.\right. \\
=\gamma\left[1-(1-\beta) L ; r p_{t}-\gamma \beta L r p_{t}+(1-L)\left(1-(1-\beta) L_{j} u_{1 t}\right.\right.
\end{array}
$$

Diferenciando o sistema formado pelas equaçōes $(7),(10)$ e (20) e resolvendo, para $r \gamma_{t^{\prime}} r p_{t}$ e $\Delta i_{t^{\prime}}$ as funçōes de transferência associadas ao modelo são obtidas:

$$
\begin{aligned}
& \left\{-(1-k L)\left[1-(1-\beta) L i^{2}+\left(\alpha_{2}+\alpha_{3}\right)\left\{\beta L-L\left[1-(1-\beta) L_{1}\right\}\right.\right.\right. \\
& \left(1-(1-\beta) L,(1-k L)-\alpha_{1} \gamma(1-L)(1-(1-\beta) L i\} r y_{t}=\right. \\
& =\left\{-\gamma(1 L)\left(1-(1-\beta) L_{3}\right\} r h_{t}-\beta^{2}(1-k) r y^{n}-\right. \\
& -\gamma(1-L)\left(1-(1-\beta) L, v_{1 t}-\left\{\left(1-(1-\beta) L j-\left(\alpha_{2}+\alpha_{3}\right)\right.\right.\right. \\
& \{\beta I .-L(1-(1-\beta) L,\}\} v_{2+}+\gamma\left(\alpha_{2}+\alpha_{3}\right)(1-L) v_{3 t}
\end{aligned}
$$




$$
\begin{aligned}
& \left\{-(1-k L)\left[1-(1-\beta) L i+\left(\alpha_{2}+\alpha_{3}\right)\left\{\beta L-L\left[1-(1-\beta) L_{1}\right\}(1-k L)-\right.\right.\right. \\
& \left.-\alpha_{1} \gamma(1-L)\right\} r_{t}=\left\{-(1-k L)[1-(1-\beta) L i\} h_{t}+(1-k) \alpha_{1} \beta r y^{n}+\right. \\
& +\left\{-(1-k L)\left[1-(1-\beta) L_{j}\right\} v_{1 t}+\alpha_{1} v_{2 t}+\left\{\left(\alpha_{2}+\alpha_{3}\right)(1-k L)\right\} v_{3 t}\right. \\
& \left\{-(1-k L)\left[1-(1-\beta) L^{2}+\left(\alpha_{2}+\alpha_{3}\right)\{\beta L-L[1-(1-\beta) L]\}[1-(1-\beta) L j ;\right.\right. \\
& \left.(1-k L)-\alpha_{1} \gamma(1-L)[1-(1-\beta) L]\right\} \Delta_{t}=\{-(1-k L)[1-(1-\beta) L] \\
& \{\beta L-L[1-(1-\beta) L]\}\} r_{t}+\left\{-(1-k L)\left[1-(1-\beta) L_{1}\right.\right. \\
& \{\beta L-L[1-(1-\beta) L j\}\} v_{1 t}+\left\{\alpha_{1}\{\beta L-L[1-(1-\beta) L j\}\} v_{2 t}+\right. \\
& +\left\{\alpha_{1} \gamma(1-L)+(1-k L)[1-(1-\beta) L j\} v_{3 t}\right.
\end{aligned}
$$

A forma estrutural do modelo com persistência no hiato do produto real tem como implicações as seguintes ordens para os polinômios de suas funções de transferência:

\begin{tabular}{lccc}
\hline Variável & AR & MA para rh & MA para o erro \\
\hline $\mathrm{ry}_{\mathrm{t}}$ & 4 & 2 & 4 \\
$\mathrm{rp}$ & 3 & 2 & 3 \\
$\Delta \mathrm{i}_{\mathrm{t}}$ & 4 & 4 & 5 \\
\hline
\end{tabular}

Supondo que a variável $r h_{t}$ é puramente exógena, pode-se representá-la pelo processo ${ }^{\prime} \mathrm{p} \mathrm{rh}_{\mathrm{t}}=\theta_{\mathrm{q}}(L) u_{5 \mathrm{t}}$. Para se chegar às equações finais a partir das funções de transfe rência acima, deve-se multiplicar $(21)$, (22) e (23) por ( $L$ ) e substituir o process
ria nas equações resul tantes.

Os graus dos polinômios no operador de defasagens resultantes deste processo são os seguintes:

\begin{tabular}{lcc}
\hline Variável & AR & NiA \\
\hline$r y_{t}$ & $p+4$ & $\max (2+q ; 2+p)$ \\
$r p_{t}$ & $p+3$ & $\max (2+q ; 2+p)$ \\
$\Delta i_{t}$ & $p+4$ & $\max (4+q ; 4+p)$ \\
$r h_{t}$ & $p$ & $q$ \\
\hline
\end{tabular}


3.3 - Um modelo com expectativas racionais e persistência no produto real

Este modelo mantém a idéia da persistência no hiato do produto real e introduz a hipótese de expectativas racionais. A estrutura reproduz a da seção anterior, i.e., equaçōes ( 2 ), ( 3 ), (4), (6) e (19), acrescentando-se a equação que descreve o processo de formação de expectativas:

$$
p_{t}^{*}=E_{t}\left(p_{t} \mid \Omega_{t}\right)
$$

onde:

$E_{t} \equiv$ operador de expectativas condicionadas no periodo $t$

$\Omega_{t} \equiv$ conjunto de informações no periodo $t$.

Em primeiro lugar deve-se eliminar a variável não-observável mas, neste caso, é necessário resolver o sistema para $\mathrm{p}_{\mathrm{t}}$ como função das variáveis pré-determinadas e dos erros. Igualando (2) e (4) de acordo com a condição de equilíbrio no mercado monetário dada por ( 6 ), e usando (3) para eliminar $i_{t}$, deriva-se uma demanda agregada no mercado de bens e serviços.

$$
p_{t}+\alpha_{1} v_{t}-\left(\alpha_{2}+\alpha_{3}\right)\left[\rho+E_{t}\left(p_{t}-p_{t-1}\right)+u_{3 t} j=h_{t}+\left(u_{4 t}-u_{2 t}\right)\right.
$$

Multiplicando-se $(25)$ por $(1-k L)$ e substituindo-se $(1-k L) y_{t}$ por sua expressão obtida de $(19)$, chega-se à solução para $p_{t}$ :

$$
\begin{aligned}
& p_{t}\left\{\left(1+\alpha_{1} \gamma\right)\left[1+\left(\frac{\alpha_{2}+\alpha_{3}-k}{1+\alpha_{1} \gamma}\right) L-\left(\frac{\left(\alpha_{2}+\alpha_{3}\right) k}{1+\alpha_{1} \dagger}\right) L^{2}\right\}=(1-k L) h_{t}-\right. \\
& -\alpha_{1}(1-k L) v_{t}^{n}+\left(\alpha_{2}+\alpha_{3}\right)(1-k) \rho-\alpha_{1} u_{1 t}+\left(\alpha_{2}+\alpha_{3}\right)(1-k L) u_{3 t}+ \\
& +(1-k L)\left(u_{4 t}-u_{2 t}\right)+\left[\alpha_{1} \gamma+\left(\alpha_{2}+\alpha_{3}\right)(1-k L)\right] p_{t}^{*} \Rightarrow \\
& \Rightarrow p_{t}=\left\{( 1 + \alpha _ { 1 } \gamma ) [ ( 1 - \psi _ { 1 } L ) ( 1 - \psi _ { 2 } L ] \} ^ { - 1 } \left\{(1-k L) h_{t}-\alpha_{1}(1-k L) v_{t}^{n_{t}}+\right.\right. \\
& +\left(\alpha_{2}+\alpha_{3}\right)(1-k) \rho-\alpha_{1} u_{1 t}+\left(\alpha_{2}+\alpha_{3}\right)(1-k L) u_{3 t}+(1-k L)\left(u_{4 t}-u_{2 t}\right)+ \\
& +\left[\alpha_{1} \gamma+1\right.
\end{aligned}
$$

onde: 


$$
\begin{aligned}
\psi_{1} \psi_{2} & =-\frac{\left(\alpha_{2}+\alpha_{3}\right) k}{1+\alpha_{1} \gamma} \\
\psi_{1}+\psi_{2} & =-\frac{\left(\alpha_{2}+\alpha_{3}-k\right)}{1+\alpha_{1} \gamma}
\end{aligned}
$$

Se $\psi_{1} \neq \psi_{2},(26)$ pode ser escrita da seguinte forma:

$$
\begin{aligned}
& p_{t}=\left(1+\alpha_{1} \gamma\right)^{-1}\left\{\frac{\psi_{1}}{\psi_{1}-\psi_{2}}\left(1-\psi_{1} L\right)^{-1}-\frac{\psi_{2}}{\psi_{1}-\psi_{2}}\left(1-\psi_{2} L\right)^{-1}\right\} \\
& \left\{(1-k L) h_{t}-\alpha_{1}(1-k L) y_{t}^{n}+\left(\alpha_{2}+\alpha_{3}\right)(1-k) \rho-\alpha_{1} u_{1 t}+\right. \\
& +\left(\alpha_{2}+\alpha_{3}\right)(1-k L) u_{3 t}+(1-k L)\left(u_{4 t}-u_{2 t}\right)+
\end{aligned}
$$$$
+\left[\alpha_{1} \gamma+\left(\alpha_{2}+\alpha_{3}\right)(1-k L), p_{t}^{*}\right\}=\left(1+\alpha_{1} \gamma\right)^{-1}\left\{\frac{\psi_{1}}{\psi_{1}-\psi_{2}}(1-k L) \sum_{j=0}^{\infty} \psi_{1}{ }^{j}{ }_{L}{ }_{h_{t}}-\right.
$$$$
\cdots \frac{\psi_{2}}{\psi_{1}-\psi_{2}}(1-k L) \underset{j=0}{\sum} \psi_{2}{ }^{j} L_{h_{t}}{ }^{\prime}-\alpha_{1}(1-k L)\left(\frac{\psi_{1}}{\psi_{1}-\psi_{2}} \sum_{j=0}^{\infty} \psi_{1}^{j}{ }_{L}^{j} y_{t}^{n}-\right.
$$$$
\left.-\frac{\psi_{2}}{\psi_{1}-\psi_{2}} \sum_{j=0}^{\infty} \psi_{2} j_{L} j_{\mathrm{t}}^{\mathrm{n}}\right)+\frac{\left(\alpha_{2}+\alpha_{3}\right)(1-k)}{\left(1-\psi_{1}\right)\left(1-\psi_{2}\right)} \rho-\alpha_{1}\left(\frac{\psi_{1}}{\psi_{1}-\psi_{2}} \sum_{j=0}^{\infty} \psi_{1}{ }^{j}{ }^{j} u_{1 t}-\right.
$$$$
\left.. \frac{\psi_{2}}{\psi_{1}-\psi_{2}} \sum_{\mathrm{j}=0}^{\infty} \psi_{2} \mathrm{j}_{L} \mathrm{j}_{\mathrm{u}_{1 \mathrm{t}}}\right)+\left(\alpha_{2}+\alpha_{3}\right)(1-\mathrm{kL})\left(\frac{\psi_{1}}{\psi_{1}-\psi_{2}} \sum_{\mathrm{j}=0}^{\infty} \psi_{1} \mathrm{j}_{\mathrm{L}}^{\mathrm{j}} \mathrm{u}_{3 \mathrm{t}}-\right.
$$$$
\left.-\frac{\psi_{2}}{\psi_{1}-\psi_{2}} \sum_{j=0}^{\infty} \psi_{2} j_{L^{\prime} u_{3 t}}\right)+(1-k L)\left(\frac{\psi_{1}}{\psi_{1}-\psi_{2}} \sum_{j=0}^{\infty} \psi_{1} j^{j}{ }^{j} u_{4 t}-\right.
$$$$
\left.-\frac{\psi_{2}}{\psi_{1}-\psi_{2}} \sum_{j=0}^{\infty} \psi_{2}{ }^{j} L^{j} u_{4 t}\right)-(1-k L)\left(\frac{\psi_{1}}{\psi_{1}-\psi_{2}} \sum_{j=0}^{\infty} \psi_{1} j_{L} j_{u_{2} t}-\right.
$$ 
54

REVISTA DE ECONOMETRIA

$$
\begin{gathered}
\left.-\frac{\psi_{2}}{\psi_{1}-\psi_{2}} \sum_{j=0}^{\infty} \psi_{2}{ }^{j} L^{j} u_{2 t}\right)+\left[\alpha_{1} \gamma+\left(\alpha_{1}+\alpha_{3}\right)(1-k L)\right] \\
\left(\frac{\psi_{1}}{\psi_{1}-\psi_{2}} \sum_{j=0}^{\infty} \psi_{1}^{j} L^{j} p_{t}^{*}-\frac{\psi_{2}}{\psi_{1}-\psi_{2}} \sum_{j=0}^{\infty} \psi_{2}^{j} L^{j} p_{t}^{*}\right)
\end{gathered}
$$

Aplicando-se o operador de expectativas, considerando $y_{t}^{n}$ como não estocástico, e representando $h_{t}$ pelo processo $\dot{\phi}_{p}(L) h_{t}=\theta_{q}(L) \epsilon_{t}$ ou $\left[1+{ }_{\psi}^{\prime}{ }_{p}(L) i h_{t}=\left[1+\theta^{\prime}{ }_{q}(k)\right] \epsilon_{t}\right.$, onde $\psi^{\prime}{ }_{p}(L)$ e $\theta^{\prime}{ }_{q}(L)$ são as partes homgêneas do processo ARMA que gera $h_{t}$, a equação (27) passa a ter a seguinte representação:

$$
\begin{aligned}
& E_{t} p_{t}=\left(1+\alpha_{1} \gamma\right)^{-1}\left\{\left[\frac { \psi _ { 1 } } { \psi _ { 1 } - \psi _ { 2 } } \left(\psi_{p}^{\prime}(L) h_{t}+\theta_{q}^{\prime}(L) \epsilon_{t}+\sum_{j=1}^{\infty} \psi_{j}^{j} h_{t-j}-\right.\right.\right. \\
& \left.-k \sum_{j=0}^{\infty} \psi j h_{t-1-j}\right)-\frac{\psi_{2}}{\psi_{1}-\psi_{2}}\left(-\psi_{p}^{\prime}(L) h_{t}+\theta_{q}^{\prime}(L) \epsilon_{t}+\right. \\
& \left.\left.+\sum_{j=1}^{\infty} \psi_{2} h_{t-j}-k \sum_{j=0}^{\infty} \psi_{2}^{j} h_{t-1-j}\right)\right]-\alpha_{1}(1-k L)\left(\frac{\psi_{1}}{\psi_{1}-\psi_{2}} \sum_{j=0}^{\infty} \psi_{1}^{j} y_{t-j}^{n}-\right. \\
& \begin{array}{c}
\left.-\frac{\psi_{2}}{\psi_{1}-\psi_{2}} \sum_{j=0}^{\infty} \psi_{2} y_{t-j}^{n}\right)+\frac{\left(\alpha_{2}+\alpha_{3}\right)(1-k)}{\left(1-\psi_{1}\right)\left(1-\psi_{2}\right)} \rho-\alpha{\frac{\psi_{1}}{\psi_{1}-\psi_{2}}}_{j=1}^{\infty} \psi_{1}^{j} u_{1 t-j}- \\
\left.-\frac{\psi_{2}}{\psi_{1}-\psi_{2}} \sum_{j=1}^{\infty} \psi_{2} u_{1 \mathrm{t}-\mathrm{j}}\right)+\left(\alpha_{2}+\alpha_{3}\right)\left[\frac { \psi _ { 1 } } { \psi _ { 1 } - \psi _ { 2 } } \left(\sum_{j=1}^{\infty} \psi_{1}^{j} u_{3 t-j}-\right.\right.
\end{array} \\
& \left.\left.-k \sum_{j=0}^{\infty} \psi j u_{3 t-1-j}\right)-\frac{\psi_{2}}{\psi_{1}-\psi_{2}}\left(\sum_{j=1}^{\infty} \psi \psi_{2} u_{3 t-j}-k \sum_{j=0}^{\infty} \psi j_{2} u_{3 t-1-j}\right)\right]+ \\
& +\left[\frac { \psi _ { 1 } } { \psi _ { 1 } - \psi _ { 2 } } \left(\sum_{j=1}^{\infty} \psi j u_{4 t-j}-k \sum_{j=0}^{\infty} \psi\left\{u_{4 t-1-j}\right)-\frac{\psi_{2}}{\psi_{1}-\psi_{2}}\left(\sum_{j=1}^{\infty} \psi_{2} \dot{j}_{4 t-j}-\right.\right.\right.
\end{aligned}
$$




$$
\begin{aligned}
& \left.\left.-k \sum_{j=0}^{\infty} \psi j_{2} u_{4 t-1-j}\right)\right]-\left[\frac{\psi_{1}}{\psi_{1}-\psi_{2}}\left(\sum_{j=1}^{\infty} \psi_{1}^{j} u_{2 t-j}-k \underset{j=0}{\sum} \psi_{1}^{j} u_{2 t-1-j}\right)-\right. \\
& \left.-\frac{\psi_{2}}{\psi_{1}-\psi_{2}}\left(\sum_{j=1}^{\infty} \psi_{2}^{j} u_{2 t-j}-k \sum_{j=0}^{\infty} \psi \psi_{2} u_{2 t-1-j}\right)\right]+ \\
& +\left[\alpha_{1} \gamma+\left(\alpha_{2}+\alpha_{3}\right)(1-k L)\left(\frac{\psi_{1}}{\psi_{1}-\psi_{2}} \sum_{\mathrm{j}=0}^{\infty} \psi_{1}^{j} \mathrm{E}_{\mathrm{t}-\mathrm{j}} \mathrm{p}_{\mathrm{t}-\mathrm{j}}-\right.\right. \\
& \left.\left.\frac{\psi_{2}}{\psi_{1}-\psi_{2}} \sum_{j=0}^{\infty} \psi_{2} E_{t-j} p_{t-j}\right)\right\}
\end{aligned}
$$

Somando e subtraindo $h_{t}, v_{t^{\prime}}^{n}, \epsilon_{t}, u_{1 t}, u_{2 t}, u_{3 t}$ e $u_{4 t}$ ao lado direito de (28) com o objetivo de substituir as representações de soma infinita por polinômios racionais e multiplicando a expressão resultante por

$$
\left(1-\psi_{1} L\right)\left(1-\psi_{2} L\right)\left(1+\alpha_{1} \gamma\right)
$$

tem-se:

$$
\begin{aligned}
& {\left[\left(1-\psi_{1} L\right)\left(1-\psi_{2} L\right)-\alpha_{1} \gamma-\left(\alpha_{2}+\alpha_{3}\right)(1-k L) i\left(1+\alpha_{1} \gamma\right) E_{t} p_{t}=\right.} \\
& =\left\{\left(1-\psi_{1} L\right)\left(1-\psi_{2} L\right)\left(-\epsilon_{t}\right)+(1-k L) h_{t}-\alpha_{1}(1-k L) y_{t}^{A}+\left(\alpha_{2}+\alpha_{3}\right)(1-k) \rho+\right. \\
& +\alpha_{1}\left[\left(1-\psi_{1} L\right)\left(1-\psi_{2} L\right)-1\right] u_{1 t}-\left(\alpha_{2}+\alpha_{3}\right)\left[\left(1-\psi_{1} L\right)\left(1-\psi_{2} L\right)-\right. \\
& -(1-k L) u_{3 t}-\left[\left(1-\psi_{1} L\right)\left(1-\psi_{2} L\right)-(1-k L)\right] u_{4 t}+ \\
& +\left[\left(1-\psi_{1} L\right)\left(1-\psi_{2} L\right)-(1-k L) i u_{2 t}\right\}
\end{aligned}
$$

Para que a nova forma estrutural seja obtida, deve-se eliminar a variłvel não-observável $E_{t} p_{t}$. Multiplicando a equação de oferta agregada, $(19$ ), por

$$
\left\{\left(1-\psi_{1} L\right)\left(1-\psi_{2} L\right)-\alpha_{1} \gamma-\left(\alpha_{2}+\alpha_{3}\right)(1-k L)\right\}\left(1+\alpha_{1} \gamma\right)
$$

e substituindo ( 29 ) na expressão resultante, tem-se: 


$$
\begin{aligned}
& \{(1-\psi) \\
& =-\gamma(1-k L) h_{t}+\left\{\alpha_{1} \gamma(1-k L)+(1-k L)\left(1+\alpha_{1} \gamma\right)[(1-\psi\right. \\
& \left.-\alpha_{1} \gamma-\left(\alpha_{2}+\alpha_{3}\right)(1-k L) ;\right\} v_{t}^{n}-\gamma\left(\alpha_{2}+\alpha_{3}\right)(1-k) \rho+\gamma\left(1-\psi_{1} L\right)(1-\psi \\
& +[(1-\psi \\
& -\gamma\left[\left(1-\psi_{1} L\right)(1-\psi\right. \\
& -(1-k L) j u_{3 t}+\gamma\left[\left(1-\psi_{1} L\right)\left(1-\psi_{2} L\right)-(1-k L)\right] u_{4 t}
\end{aligned}
$$

Multiplicando-se agora a equação da taxa Je juros, ( 3 ), por

$$
\left\{\left(1-\psi_{1} L\right)\left(1-\psi_{2} L\right)-\alpha_{1} \gamma-\left(\alpha_{2}+\alpha_{3}\right)(1-k L)\right\}\left(1+\alpha_{1} \gamma\right)
$$

e substituindo-se, mais uma vez, (29) no resultado obtido, vem:

$$
\begin{aligned}
& \left\{\left(1-\psi_{1} L\right)(1-\psi\right. \\
& =\left\{\left(1+\alpha_{1} \gamma\right)(1-\psi\right. \\
& +(1-k L) h_{t}-\alpha_{1}(1-k L) y_{t}^{n}+\alpha_{1}[(1-\psi \\
& +[(1-\psi \\
& -(1-k L)] u_{3 t}-\left[\left(1-\psi_{1} L\right)\left(1-\psi_{2} L\right)-(1-k L) j u_{4 t}\right.
\end{aligned}
$$

Após a eliminação da variável não-observável, completa-se a nova forma estrutural com a equação da demanda agregada:

$$
p_{t}+\alpha_{1} y_{t}=\left(\alpha_{2}+\alpha_{3}\right) i_{t}+h_{t}+\left(u_{4 t}-u_{2 t}\right)
$$

Diferenciando o sistema de equações (30), (31) e (32) e resolveindo para $r y_{t^{\prime}} r p_{t}$ e $\Delta i_{t}$, chega-se às fınções de transferência do modelo: 


$$
\begin{aligned}
& {\left[\left(1-. \psi_{1} L\right)\left(1-\psi_{2} L\right)-\alpha_{1} \gamma-\left(\alpha_{2}+\alpha_{3}\right)(1-k L)\right]\left(1+\alpha_{1} \gamma\right)[(1-k L)+} \\
& +\alpha_{1} \gamma+(1-k L) L\left(\alpha_{2}+\alpha_{3}\right) j r y_{t}=\left\{\left[1+\left(\alpha_{2}+\alpha_{3}\right) L_{j}[-\gamma(1-k L) j+\right.\right. \\
& +\gamma\left[\left(1-\psi_{1} L\right)\left(1-\psi_{2} L\right)-\gamma \alpha_{1}-\left(\alpha_{2}+\alpha_{3}\right)(1-k\right. \\
& \left.+(1-k L) \gamma\left(\alpha_{2}+\alpha_{3}\right)\right\} r h_{t}+\left\{\gamma \alpha_{1}(1-k L)+(1-k L)\left[\left(1-\psi_{1} L\right)\left(1-\psi_{2} L\right)-\right.\right. \\
& -\gamma \alpha_{1}-\left(\alpha_{2}+\alpha_{3}\right)(1-k L) ;\left(1+\alpha_{1} \gamma\right)\left[1+\left(\alpha_{2}+\alpha_{3}\right) L_{j}+\right. \\
& +\gamma\left(\alpha_{2}+\alpha_{3}\right)\left[-\alpha_{1}(1-k L)\right\} r y^{n}+\left[1+\left(\alpha_{2}+\alpha_{3}\right) L i v_{1 t}\right. \\
& +\gamma\left[\left(1-\psi_{1} L\right)\left(1-\psi_{2} L\right)-\gamma \alpha_{1}-\left(\alpha_{2}+\alpha_{3}\right)(1-k L) j\left(1+\alpha_{1} \gamma\right) v_{2 t}+\right. \\
& +\gamma\left(\alpha_{2}+\alpha_{3}\right) v_{3 t}
\end{aligned}
$$

$\left[\left(1-\psi_{1} L\right)\left(1-\psi_{2} L\right)-\gamma \alpha_{1}-\left(\alpha_{2}+\alpha_{3}\right)(1-k L) ;\left(1+\alpha_{1} \gamma\right)[(1-k L)+\right.$$$
+\gamma \alpha_{1}+(1-k L) L\left(\alpha_{2}+\alpha_{3}\right) ; r p_{t}=\left\{\gamma \alpha_{1}(1-k L)+\left(\left(1-\psi_{1} L\right)\left(1-\psi_{2} L\right)-\right.\right.
$$$$
\text { - } \left.\gamma \alpha_{1}-\left(\alpha_{2}+\alpha_{3}\right)(1-k L) ;\left(1+\alpha_{1} \gamma\right)(1-k L)+(1-k L)^{2}\left(\alpha_{2}+\alpha_{3}\right)\right\} \mathrm{rh}_{\mathrm{t}}-
$$$$
-\left\{\alpha _ { 1 } \left\{\alpha_{1} \gamma(1-k L)+(1-k L)\left[\left(1-\psi_{1} L\right)\left(1-\psi_{2} L\right)-\alpha_{1} \gamma-\right.\right.\right.
$$$$
\left.\left.-\left(\alpha_{2}+\alpha_{3}\right)(1-k L) ;\left(1+\alpha_{1} \gamma\right)\right\}+\alpha_{1}(1-k L)(1-k L)\left(\alpha_{2}+\alpha_{3}\right)\right\} r y^{n}-
$$$$
-\alpha_{1} v_{1 \mathrm{t}}+\left[\left(1-\psi_{1} L\right)\left(1-\psi_{2} \mathrm{~L}\right)-\alpha_{1} \gamma-\left(\alpha_{2}+\alpha_{3}\right)(1-k L) i\left(1+\alpha_{1} \gamma\right)(1-k L) v_{2 t}+\right.
$$$$
+(1-k L)\left(\alpha_{2}+\alpha_{3}\right) v_{3 t}
$$

$$
\begin{aligned}
& {\left[\left(1-\psi_{1} L\right)\left(1-\psi_{2} L\right)-\gamma \alpha_{1}-\left(\alpha_{2}+\alpha_{3}\right)(1-k L) i\left(1+\alpha_{1} \gamma\right)\left[(1-k L)+\gamma \alpha_{1}+\right.\right.} \\
& +(1-k L) L\left(\alpha_{2}+\alpha_{3}\right) \cdot \Delta i_{t}=\left\{-\alpha_{1} \gamma(1-k L) L-\left[\left(1-\psi_{1} L\right)\left(1-\psi_{2} L\right)-\right.\right. \\
& -\gamma \alpha_{1}-\left(\alpha_{2}+\alpha_{3}\right)(1-k L) ;\left(1+\alpha_{1} \gamma\right)(1-k L) L+(1-k L)\left[\alpha_{1} \gamma+(1-k L) j\right\} r h_{t}+ \\
& +\left\{\alpha_{1}^{2} \gamma(1 k L)+(1-k L)\left[\left(1-\psi_{1} L\right)\left(1-\psi_{2} L\right)-\alpha_{1} \gamma-\right.\right.
\end{aligned}
$$




$$
\begin{aligned}
& \left.\cdots\left(\alpha_{2}+\alpha_{3}\right)(1-k L) j\left(1+\alpha_{1} \gamma\right) \alpha_{1}-\alpha_{1}(1-k L)\left[(1-k L)+\gamma \alpha_{1}\right]\right\} r y^{n}+ \\
& +\alpha_{1} L v_{1 t}-(1-k L) L\left[\left(1-\psi_{1} L\right)\left(1-\psi_{2} L\right)-\gamma \alpha_{1}-\right. \\
& -\left(\alpha_{2}+\alpha_{3}\right)(1-k L),\left(1+\alpha_{1} \gamma\right) v_{2 t}+\left[(1-k L)+\alpha_{1} \gamma i v_{3 t}\right.
\end{aligned}
$$

As ordens dos polinômios nas funções de transferências são:

\begin{tabular}{lccc}
\hline Variável & AR & MA para rh & MA para o erro \\
\hline$r y_{t}$ & 4 & 2 & 4 \\
$r p_{t}$ & 4 & 3 & 4 \\
$\Delta i_{t}$ & 4 & 4 & 5 \\
\hline
\end{tabular}

Para se passar às equações finais, deve-se substituir o processo estocástico independente da variável $r h_{t^{\prime}}$

$$
\mu_{p-1}(L) r_{t}=\theta_{q}(L) \epsilon_{t}(1) \text {. }
$$

nas funções de transferência.

As ordens esperadas para as equações finais são:

\begin{tabular}{lcc}
\hline Variável & AR & $M A$ \\
\hline$r y_{t}$ & $3+p$ & $\max (2+q ; 3+p)$ \\
$r p_{t}$ & $3+p$ & $\max (3+q ; 3+p)$ \\
$\Delta i_{t}$ & $3+p$ & $\max (4+q ; 4+p)$ \\
$r h_{t}^{t}$ & $p-1$ & $q$ \\
\hline
\end{tabular}

(1)

Admitindo-se que as estacionariedade da série do logaritmo da base monetária em termos nominais seja alcançada na $d$-ésima diferença, pode-se escrever

$$
\lambda_{p-d}(L)(1-L)^{d} h_{t}=\theta_{q}(L) \epsilon_{t} .
$$

Tem-se, pois, que

$$
\phi_{p}(L)=\lambda_{p-d}(L)(1-L)^{d}
$$

e que

$$
\mu_{p-1}(L)=\lambda_{p-d}(L)(1-L)^{d-1}
$$


3.4 - Um modelo generalizado com expectativas racionais

Nesta seção, vários polinômios no operador de defasagens são introduzidos no modelo neoclássico até então considerado, com o objetivo de levar em consideração a possibilidade de estruturas de defasagens mais complexas no processo de oferta monetária, nos ajustamentos da demanda por moeda e na resposta da atividade econômica à inflação não-antecipada. A esta nova estrutura incluimos as equações ( 3 ), (6) e (24).

$$
\begin{aligned}
& \phi_{1}(L)\left(y_{t}-y_{t}^{n}\right)=\gamma\left(p_{t}-p_{t}^{*}\right)+u_{1 t} \\
& \phi_{2}(L)\left(m_{t}^{d}-p_{t}\right)=\alpha_{1} \psi_{3}(L) y_{t}-\alpha_{2} \psi_{4}(L) i_{t}+u_{2 t} \\
& \phi_{5}(L) m_{t}^{s}=\psi_{6}(L) h_{t}+\alpha_{3} \psi_{7}(L)+u_{4 t}
\end{aligned}
$$

A solução para $p_{t}$ é obtida quando se iguala (37) e (38), de acordo com $(6)$, e se substitui a expressão de $i_{t}$, dada por ( 3 ), na equação resultante:

$$
\begin{aligned}
& \mathrm{p}_{\mathrm{t}}\left[\mathrm{L}\left(\alpha_{2} \phi_{4}(\mathrm{~L})+\alpha_{3} \phi_{2}(\mathrm{~L}) \psi_{5}^{-1}(\mathrm{~L}) \psi_{7}(\mathrm{~L})\right)+\psi_{2}(\mathrm{~L})+\alpha_{1} \gamma \psi_{1}^{-1}(\mathrm{~L}) \psi_{3}(\mathrm{~L})\right]= \\
& =\left[\psi_{2}(\mathrm{~L}):_{5}^{-1}(\mathrm{~L}) \psi_{6}(\mathrm{~L}) j \mathrm{~h}_{\mathrm{t}}+\left[\alpha_{2} \psi_{4}(\mathrm{~L})+\alpha_{3} \psi_{2}(\mathrm{~L}) \dot{\phi}_{5}^{-1}(\mathrm{~L}) \psi_{7}(\mathrm{~L}) j \rho+\right.\right. \\
& +\left[\alpha_{1} \gamma \psi_{1}^{-1}(\mathrm{~L}) \phi_{3}(\mathrm{~L})+\alpha_{2} \psi_{4}(\mathrm{~L})+\alpha_{3} \psi_{2}(\mathrm{~L}) \psi_{5}^{-1}(\mathrm{~L}) \psi_{7}(\mathrm{~L})\right] \mathrm{p}_{\mathrm{t}}^{*}+ \\
& +\left[\alpha_{2} \phi_{4}(\mathrm{~L})+\alpha_{3} \psi_{2}(\mathrm{~L}) \psi_{5}^{-1}(\mathrm{~L}) \psi_{7}(\mathrm{~L}) j u_{3 \mathrm{t}}+\psi_{2}(\mathrm{~L}) \psi_{5}^{-1}(\mathrm{~L}) \mathrm{u}_{4 \mathrm{t}}-\right. \\
& -\alpha_{1} \phi_{3}(\mathrm{~L}) y_{\mathrm{t}}^{\mathrm{n}}-\alpha_{1} \psi_{3}(\mathrm{~L}) \phi_{1}^{-1}(\mathrm{~L}) \mathrm{u}_{1 \mathrm{t}}-\mathrm{u}_{2 \mathrm{t}}
\end{aligned}
$$

Com o intuito de simplificar a álgebra sem incorrer, no entanto, em perda de generalidade, ( 2 ) supõe-se que ${ }^{\prime 2} 2, \psi_{4}, \psi_{5}, \psi_{6}, \psi_{7}, \alpha_{1}$ e $\alpha_{2}$ sejam parâmetros constantes e que $\psi_{1}(L) \propto_{\psi_{3}}(L)$. Assim sendo, a equação $\left(39^{2}\right)$ pode ser reescrita da seguinte maneira:

\section{(2)}

De acordo com Quenouille, as variáveis endógenas nas equaçōes finais possuem polinômios autoregressivos idênticos. Desta forma, $\mathrm{ry}_{\mathrm{t}}, \mathrm{rp}_{\mathrm{t}}$ e $\Delta_{\mathrm{t}}$ comportam-se de maneira análoga durante os ciclos econômicos. Como ficará claro mais adiante, quando da obtenção das ordens esperadas para as funções de transferência e equações finais, qualquer que seja o grau dos polinômios AR verificado empiricamente, este pode ser explicado por uma ordem de $\psi_{1}(\mathrm{~L})$ suficientemente alta. 


$$
\left(\beta_{0}-\psi L\right) p_{t}=\beta_{1} h_{t}+\beta_{2}+\beta_{3} p_{t}^{*}+\beta_{4} u_{3 t}+\beta_{5} u_{4 t}-\beta_{6}(L) v_{t}^{n}-\beta_{7} u_{1 t}-u_{2 t}
$$

onde

$$
\begin{aligned}
& \beta_{0} \equiv\left[r_{2}+\alpha_{1} \gamma_{1}-\gamma_{1} L\right) \psi_{3}(L) \\
& \psi \quad \equiv \quad-\left\{\alpha_{2} r_{4}+\alpha_{3} \psi_{2} r_{5}^{-1} \psi_{7}\right) \\
& \beta_{1} \equiv r_{2}^{-1}{ }^{-1} \\
& \beta_{2} \equiv-\psi \rho \\
& \beta_{3} \equiv\left(\alpha_{1} \gamma \psi_{1}^{-1}(L) \cdot \psi_{3}(L)+\alpha_{2} \phi_{4}+\alpha_{3} \psi_{2} \psi_{5}^{-1} \psi_{7}\right) \\
& \beta_{4} \equiv-\psi \\
& \beta_{5} \equiv \psi_{2} \cdot{ }^{-1} \\
& \beta_{6}(L) \equiv \alpha_{1}+3(L) \\
& \beta_{7}=\alpha_{1 * i}{ }^{1}(L) * 3(L)
\end{aligned}
$$

Supondo que a trajetória de $p_{t}$ satisfaça a condição de estabilidade, isto é, , $\psi:<1$, tem-se a seguinte representação para $(40)$ :

$$
\begin{aligned}
& p_{t}=\beta_{0}^{-1}\left[\beta \sum_{j=0}^{\infty}\left(\psi / \beta_{0}\right)^{j} h_{t-j}+\beta_{2}\left[1-\left(\psi / \beta_{0}\right)_{1}+\beta_{3} \sum_{j=0}^{\infty}\left(\psi / \beta_{0}\right)^{j} p_{t-j}^{*}+\right.\right. \\
& +\beta_{4} \sum_{j=0}^{\infty}\left(\psi / \beta_{0}\right)^{j} u_{3 t-j}+\beta_{5} \sum_{j=0}^{\infty}\left(\psi / \beta_{0}\right)^{j} u_{4 t-j}-\beta_{6}(L) \sum_{j=0}^{\infty}\left(\psi / \beta_{0}\right)^{j} y_{t-j}^{n}- \\
& \left.-\beta_{7} \sum_{j=0}^{\infty}\left(\psi / \beta_{0}\right)^{j} u_{1 t-j}-\sum_{j=0}^{\infty}\left(\psi / \beta_{0}\right)^{j} u_{2 t-j}\right]
\end{aligned}
$$

Aplicando-se o operador de expectativas, considerando $y_{t}^{n}$ como não estocástico e representando $h_{t}$ pelo processo $\sim p(L) h_{t}=\theta_{q}(L) \epsilon_{t^{\prime}}$ ou $\left[1+\phi_{p}^{\prime}(L), h_{t}=\left[1+\theta_{q}^{\prime}(L), \epsilon_{t}\right.\right.$, onde $p_{p}^{\prime}(L)$ e $\theta_{q}^{\prime}(L)$ são as partes homo- 
gèneas do processo ARMA que gera $h_{t}$, tem-se:

$$
\begin{aligned}
& E_{t} p_{t}=\beta_{0}^{-1}\left\{\beta _ { 1 } \left[-r^{\prime} p\right.\right. \\
& \infty \\
& j=1 \\
& +\beta_{2}\left[1-\left(\psi / \beta_{0}\right) . \quad j=0 \quad j=1\right. \\
& +\beta_{5} \sum_{j=1}^{\infty}\left(\psi / \beta_{0}\right) \quad \sum_{j=0}^{\infty}\left(\psi / \beta_{0}\right)^{j} y_{t-j}^{n}- \\
& -\beta_{7} \underset{j=1}{\infty}\left(\psi / \beta_{0}\right)^{j} u_{1 t-j}-\sum_{j=1}^{\infty}(\psi
\end{aligned}
$$

Somando e subtraindo $h_{t^{\prime}}, y_{t^{\prime}}^{n} \epsilon_{t^{\prime}}, u_{1 t^{\prime}}, u_{2 t^{\prime}} u_{3 t}$ e $u_{4 t}$ ao lado direito de (42) com o objetivo de substituir as representações de soma infinita por polinômios racionais, multiplicando a expressão resultante por $\left(\beta_{0}-\psi L\right)$ e coletando os termos comuns, tem-se:

$$
\begin{aligned}
& {\left[\left(\beta_{0}-\beta_{3}\right)-\psi L . E_{t} p_{t}=\beta_{1} h_{t}-\beta_{1}\left[1-\left(\psi / \beta_{0}\right.\right.\right.} \\
& +\beta_{5}\left(\psi / \beta_{0}\right) L u_{4 t}-\beta_{6}(L) y_{t}^{n}-\beta_{7}\left(\psi / \beta_{0}\right) L u_{1 t}-\left(\psi / \beta_{0}\right)
\end{aligned}
$$

A nova forma estrutural para $y_{t}, p_{t}$ e $i_{t}$ pode ser obtida eliminando-se a variável não-observável $E_{t} p_{t}$ no sistema inicial. Multiplicando-se (36) por $\left[\left(\beta_{0} \cdot \beta_{3}\right)-\psi L\right.$. oferta agregada como função, apenas, das variáveis observáveis:

$$
\begin{aligned}
& \phi_{1}(L)\left[\left(\beta_{0}-\beta_{3}\right)-\psi L\right. \\
& =-\gamma \beta_{1} h_{t}+\left\{\gamma \beta_{6}(L)+,_{1}(L)\left[\left(\beta_{0}-\beta_{3}\right)-\psi L \mathrm{j}\right.\right. \\
& +\gamma \beta_{1}\left[1-\left(\psi / \beta_{0}\right)\right. \\
& +\left[\left(\beta_{0}-\beta_{3}\right)-\psi L+\gamma \beta_{7}\left(\psi / \beta_{0}\right) L\right] u_{1 t}+\gamma(\psi
\end{aligned}
$$


Multiplicando-se (37) por $\psi_{5}$ e (38) por $\psi_{2}$ e igualando-se as expressões obtidas, chega-se à equação da demanda agregada:

$$
\begin{aligned}
& \phi_{2} \phi_{5} p_{t}+\alpha_{1 \psi_{3}}(\mathrm{~L})_{\psi_{5}} y_{\mathrm{t}}-\left(\alpha_{2} \psi_{4} \psi_{5}+\alpha_{3} \psi_{2} \psi_{7}\right) i_{\mathrm{t}}= \\
& =\phi_{2} \phi_{6} h_{\mathrm{t}}+\left(\phi_{2} \mathrm{u}_{4 \mathrm{t}}-\psi_{5} \mathrm{u}_{2 \mathrm{t}}\right)
\end{aligned}
$$

Multiplicando (3) por $\left[\left(\beta_{0}-\beta_{3}\right)-1\right.$

resultado, obtém-se a equação para a taxa nominal de juros, em função apenas das variáveis observáveis:

$$
\begin{aligned}
& {\left[\left(\beta_{0}-\beta_{3}\right)-i\right.} \\
& +\beta_{2}-\beta_{6}(L) y_{t}^{n}+\{[(\beta \\
& -\beta_{7}(?
\end{aligned}
$$

As equações ( 44 ), ( 45 ) e (46) descrevem a forma estrutural do modelo após a eliminação da variável não-observável $E_{t} p_{t}$.

Diferenciando este sistema e resolvendo para $r y_{t}, r p_{t}, \Delta i_{t}$, obtem-se as funções de transferência do modelo:

$$
\begin{aligned}
& {\left[\left(\beta_{0}\right.\right.} \\
& =\left\{-\gamma \beta_{1}\left[r_{2}\right.\right. \\
& +\gamma \\
& \text { 1. } 2 \psi_{5}+\alpha_{2}+4 \cdot 5 \\
& +\left[r_{2} \cdot 5+\left(\alpha_{2} \phi_{4} \psi_{5}+\alpha_{3} \psi_{2} r_{7}\right)\right. \\
& +\gamma\left(\alpha_{2} *_{4} \cdot v_{5}+\alpha_{3 * 2 * 7}\right) v_{3 t} \\
& =\left\{\alpha_{1}: 3^{\prime}(L)\right. \\
& \left.\left.+\alpha_{3} p_{2}: p_{7}\right)\right\} n_{t}-\left\{\alpha_{1} \psi_{3}(1) \psi_{5}[\gamma\right.
\end{aligned}
$$




$$
\begin{aligned}
& \left.+\beta_{6}(1) r v^{n}{ }_{\nu_{1}}(1)\left(\alpha_{2} r_{4} \varphi_{5}+\alpha_{3} \psi_{2} \psi_{7}\right)\right\}-\alpha_{1} \psi_{3}(\mathrm{~L})_{\psi_{5} v_{1 \mathrm{t}}}+
\end{aligned}
$$

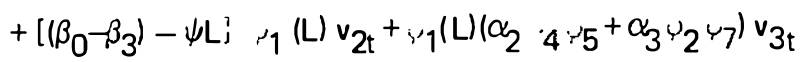

$$
\begin{aligned}
& {\left[\left(\beta_{0^{-}}-\beta_{3}\right)-\psi L_{1}\left[\psi_{1}(L) ;{ }^{\psi_{1}}{ }_{5}+\alpha_{1} \gamma_{\psi_{3}}(L) \psi_{5}+,_{1}(L)\left(\alpha_{2} \phi_{4} \psi_{5}+\right.\right.\right.}
\end{aligned}
$$

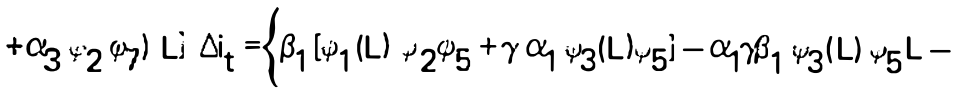

$$
\begin{aligned}
& \left.-,_{1}(L){ }_{\gamma_{2} \varphi_{6}}\left[\left(\beta_{0}-\beta_{3}\right)-\psi L\right] L\right\} h_{t}+\left\{\left[\gamma_{6}(1)+{ }_{\psi_{1}}(1)\left(\beta_{0}-\beta_{3}-\psi\right)\right] r y^{n} \alpha_{1} \cdot \mu_{3}(L)\right. \\
& \psi_{5} \mathrm{~L}-\beta_{6}(1) r y^{\mathrm{n}}\left[\psi_{1}(1), r_{2} \psi_{5}+\gamma \alpha_{1} \iota_{3}(1)_{\psi_{5}}^{j}\right\}+\alpha_{1} \psi_{3}(\mathrm{~L})_{\psi_{5}} \mathrm{~L} v_{1 \mathrm{t}}- \\
& -\psi_{1}(L)\left[\left(\beta_{0}-\beta_{3}\right)-\psi L, L v_{2 t}+\left[\psi_{1}(L):_{2} \psi_{5}+\gamma \alpha_{1} \psi_{3}(L) \psi_{5}\right] v_{3 t}\right.
\end{aligned}
$$

A forma estrutural do modelo tem como implicações as seguintẹs ordens para os polinômios das funções de transferência:

\begin{tabular}{cccc}
\hline Variável & AR & MA para $\mathrm{rh}_{\mathrm{t}}$ & MA para os erros \\
\hline $\mathrm{ry}_{\mathrm{t}}$ & $2+\mathrm{ORD}_{\psi_{1}}(\mathrm{~L})$ & 1 & 3 \\
$\mathrm{rP}_{\mathrm{t}}$ & $2+\mathrm{ORD}_{\psi_{1}}(\mathrm{~L})$ & $1+\mathrm{ORD}_{1}(\mathrm{~L})$ & $2+\mathrm{ORD}_{\psi_{1}}(\mathrm{~L})$ \\
$\mathrm{\Delta i}_{\mathrm{t}}$ & $2+\mathrm{ORD}_{\psi_{1}}(\mathrm{~L})$ & $2+\mathrm{ORD}_{\psi_{1}}(\mathrm{~L})$ & $3+\mathrm{ORD}_{\psi_{1}}(\mathrm{~L})$ \\
\hline
\end{tabular}

Com base na hipótese de que o logaritmo da base monetária em termos nominais é não estacionário, o processo $\operatorname{ARMA} \varphi_{p}(L) h_{t}=\theta_{q}(L) \epsilon_{t}$ pode ser representado por $\iota_{p-1}(L)(1-L) h_{t}=\theta_{q}(L) \epsilon_{t}$. Multiplicando $(479$, ( 48$)$ e ( 49 ) por $\mu_{p-1}(L)$ e substituindo-se em cada uma destas equações o processo estocástico

\begin{tabular}{|c|c|c|}
\hline Variável & $A R$ & MA \\
\hline $\begin{array}{l}r y_{t} \\
r p_{t} \\
\Delta i_{t} \\
r h_{t}\end{array}$ & $\begin{array}{l}p+1+\text { ORD } \varphi_{1}(L) \\
p+1+\text { ORD } 1(L) \\
p+1+\text { ORD } y_{1}(L) \\
p-1\end{array}$ & $\begin{array}{c}\max (q+1 ; p+2) \\
\max \left[q+1+O R D \psi_{1}(L) ; p+1+O R D \psi_{1}(L) j\right. \\
\max \left[q+2+O R D \psi_{1}(L) ; p+2+O R D \psi_{1}(L) j r h\right. \\
q\end{array}$ \\
\hline
\end{tabular}
da variável exógena, chega-se às equações finais do modelo.

As ordens esperadas para as equações finais são: 


\section{1.- ANÁLISE EMPIRICA DAS EQUAÇÕES FINAIS E DAS FUNÇÕES DE TRANSFERENNCIA}

As formas estruturais dos modelos monetários desenvolvidos na seção 3 têm como implicações testáveis as or dens das funções de transferência e modelos ARMA para produto real, taxa de juros e inflação. Nesta seção aplicam-se aos dados brasileiros as técnicas de identificação e estimação sugeridas por Box e Jenkins, com o objetivo de identificar os modelos cujas implicações são compative is com as informações contidas nos dados e, conseqüentemente, as hipóteses econô. micas que não são rejeitadas pela evidência empirica no Brasil.

A análise foi desenvolvida em bases mensais $(1975-80)$ e trimestrais (1971-80) para as equações finais e, também, em bases semestrais (1975-80, 72 observações, considerando-se o semestre que termina a cada mês) para as funções de transferência.

A produção industrial foi considerada como "proxy" para o produto real, e entre as diversas alternativas de taxas de juros, foram utilizadas as taxas de juros das financeiras (crédito ao consumidor) que representam o segmento mais livre de um mercado altamente controlado. (3)

No processo de identificação das equações finais foram examinadas as funções de autocorrelação e de autocorrelação parcial (respectivamente AC e $A C P$ I, considerando-se os processos ARMA alternativos sugeridos pelo comportamento destas funções. Para a comparação destes processos, foi empregado o teste da razão de verossimilhança ( $R V$ ), a fim de evitar o caráter subjetivo dos métodos de Box e Jenkins (Zellner e Palm, 1974, pág. 32).

Serão discutidas, primeiramente, as estimativas efetuadas em bases mensais. A Tabela 1 apresenta os resultados obtidos para o logaritmo do nivel de preços. Rejeita-se de início o modelo $\mathrm{m}_{2}$, devido à não-significância de seus coeficientes e ao resultado do teste $F$. $O$ teste $R V$ indica que os modelos $m_{4}$ e $m_{5}$ são mais adequados que $m_{3}$. O mesmo teste não pode ser feito com o modelo $m_{1}$, uma vez que os parâmetros deste não representam um subconjunto dos parâmetros dos modelos $(1,2,2)$. Por esta razão, o modelo $m_{1}$ será considerado, juntamente com $m_{4}$ e $m_{5}$, como um dos processos estocásticos capazes de descrever a série do nível de preços em sua forma logarítmica.

\section{(3)}

As fontes dos dados utilizados sāo:

(i) nivel de preços: IGP, Col. 2, Conjuntura Econômica, FGV;

(ii) produçāo industrial: Indústria de Transformaçāo; Indicadores Conjunturais da Indústria, IBGE;

(iii) taxas de juros: Taxas de Juros das Financeiras no Rio de Janeiro (Crédito ao Consumidor), Quadro I.28, Boletim do Banco Central do Brasil;

(iv) base monetária: Quadro I.1, Boletim do Banco Central do Brasil. 
TABELA 1

LN DO NIVEL DE PREÇOS, dados mensais, 1975-80: equações finais estimadas e testes da razão de verossimilhança

\begin{tabular}{|c|c|c|c|c|c|c|c|c|c|c|c|}
\hline MODELOS & $S_{Q R}^{(a)}$ & $\mathrm{GL}^{(\mathrm{b})}$ & SQR/GL & $\overline{\mathrm{AR}_{1}}$ & $\mathrm{AR}_{2}$ & CONSTANTE & $\mathrm{MA}_{2}$ & $\overline{\mathrm{R}}^{2}$ & $Q(12)^{(c)}$ & $Q(1.2)^{(c)}$ & $\mathbf{F}$ \\
\hline $\mathrm{m}_{1}:(2,2,2)$ & 0,00597 & 67 & 0,000089104 & & $\begin{array}{l}-0,26715 \star \\
(-2,014)\end{array}$ & $\begin{array}{r}0,00083 \\
(1,1311) \\
\end{array}$ & $\begin{array}{l}0,45075 \star \\
(3,706)\end{array}$ & 0,168 & $\begin{array}{l}6,5 * \star \\
(9 \mathrm{df}) \\
\end{array}$ & $\begin{array}{r}9,9 * \star \\
(21 \mathrm{df}) \\
\end{array}$ & $\begin{array}{l}F(2,67)= \\
x \quad 7,95 \star \star \star\end{array}$ \\
\hline $\mathrm{E}_{3}:(2,2,2)$ & 0,00587 & 66 & 0,00008894 & $\begin{array}{l}-0,46183 \star \\
(-3,779)\end{array}$ & $\begin{array}{l}0,18863 * \\
(0,8481)\end{array}$ & $\begin{array}{l}0,00083 \\
(1,669)\end{array}$ & $\begin{array}{l}0,6146 \star \\
(3,027)\end{array}$ & 0,168 & $\begin{array}{l}5,8 * \star \\
(8 \mathrm{df}) \\
\end{array}$ & $\begin{array}{r}9,1^{\star \star} \\
\left(20^{d f}\right) \\
\end{array}$ & $\begin{array}{l}F(3,66) \\
=5,65 \star \star \star\end{array}$ \\
\hline$a_{4}:(1,2,2)$ & 0,00592 & 67 & 0,000088358 & $\begin{array}{l}-0,45030 * \\
(-3,646)\end{array}$ & & $\begin{array}{l}0,00096 \\
(1,524)\end{array}$ & $\begin{array}{l}0,46363 \star \\
(3,793)\end{array}$ & 0,175 & $\begin{array}{r}6,5 * \star \\
(19 \mathrm{df}) \\
\end{array}$ & $\begin{array}{l}10,1 \star \star \\
(21 \mathrm{df}) \\
\end{array}$ & $\begin{array}{l}F(2,67) \\
=8,31 \leqslant * \pi\end{array}$ \\
\hline $\mathrm{w}_{5}:(1,2,2)$ & 0.00609 & 68 & 0,000089559 & $\begin{array}{l}-0,39179 \star \\
(-3,208)\end{array}$ & & & $\begin{array}{l}0,40234 \star \\
(3,331)\end{array}$ & 0,149 & $\begin{array}{r}6,5 * \pi \\
(10 \mathrm{df}) \\
\end{array}$ & $\begin{array}{c}10,2 \\
(22 \text { of })\end{array}$ & $\begin{array}{l}F(2,68)= \\
=7,16 \star \star \star\end{array}$ \\
\hline MDELOS COMPARADOS & & & $\lambda=\frac{L\left(X H_{1}\right)}{L\left(X H_{0}\right)}$ & & & $2 \ln \lambda$ & & r & & & \\
\hline $\mathrm{H}_{0}: \mathrm{m}_{4}$ vs $\mathrm{H}_{3}: \mathrm{m}_{3}$ & & & 1,35709 & & & 0,61069 & & 1 & & & \\
\hline $\mathrm{H}_{0}: \mathrm{m}_{5}$ vs $\mathrm{H}_{1}: \mathrm{m}_{3}$ & & & 3,76054 & & & 2,64913 & & 2 & & & \\
\hline
\end{tabular}

(a) - Sorı dos Quadrados dos Resíduos

(b) - Graus de Liberdade

(c) - Estatística Box-Pierce (distribuição qui-quadrada), que testa a significância de um conjunto de correlações amostrais para as defasagens, respectivamente, de 1 a 12 e de 1 a 24

(d) - Os valores entre parênteses são as estatísticas $t$ dos coeficientes estimados

* - indica signnificância estatística ao nível de $5 \pi$ (distribuição $t$ )

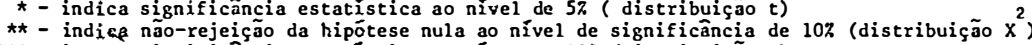

$\star \star \star$ - indica significância estatística ao nível de $10 \%$ (distribuição $F$ )

,,++++++- indicam respectivamente rejeição da hipótese nula aos niveis de significância de $5 \%$, $10 \%$ e $25 \%$ (2 ln $\lambda$ possui distribuição $x^{2}$ com $r$ graus de liberdade) 
Os resultados da análise dos dados de produção industrial são descritos na Tabela 2. Os padrões de comportamento das funções $A C$ e $A C P$ sugerem que o processo $(2,1,1) \times(0,1,0) 12$ pode representar uma boa aproximação, embora esquemas mais simples como $(0,1,1) \times(0,1,0)_{12},(2,1,0) \times(0,1,0)_{12}$ ou $(1,1,1) \times(0,1,0) 12$ também sejam compativeis com aquelas funções.

Os coeficientes AR mostraram-se estatisticamente não significantes quando estimados separadamente (modelos $m_{4}$ e $m_{5}$ ). o mesmo acontecendo quando considerados em conjunto com o termo MA (modelo $\mathrm{m}_{6}$ ). Quando se abandona o componente de média móvel (modelo $\mathrm{m}_{3}$ ) os termos autoregressivos tornam-se significantes, enquanto o modelo $M A$ puro $\left(\mathrm{m}_{2}\right)$ também apresenta um bom ajustamento. Estes resultados, que indicam um melhor desempenho dos modelos $A R$ ou MA, são confirmados pelos testes RV nos quais os modelos mistos são rejeitados.

A Tabela 3 resume os modelos estimados para as taxas de juros. A função $A C$ apresenta valores estatisticamente diferentes de zero para as defasagens 1,2 e 3, o mesmo acontecendo para a função ACP para as defasagens 1 e 4 . Partindo-se de um esquema puramente $M A\left(m_{1}\right)$ e introduzindo-se termos autoregressivos, o modelo $\mathrm{m}_{4}$ emerge como o processo que melhor reproduz a série original, o que pode ser visto também pelo resultado do teste RV.

A série de base monetária, em temos logarítimicos, segue um processo do tipo "passeio aleatório" com um termo MA sazonal, já que todos os valores da função $A C$, exceto para a defasagem 12, mostraram-se estatisticamente nulos. Os resultados das estimações são apresentados na Tabela 4.

Discutem-se, em seguida, as análises trimestrais, sendo apresentados na Tabela 5 os modelos estimados para o logaritmo do nivel de preços. A comparação entre $m_{1}$ e $m_{4}$ é favorável ao primeiro modelo: o termo MA de grau.um introduzido em $m_{4}$ não se mostrou significante e o teste RV confirmou a superioridade de $m_{1}$. Este mesmo teste aponta $m_{3}$ como uma representação mais adequada que $m_{2}$, apesar da não-significância do termo $A R$ e do termo $M A$ de primeiro grau, o que parece sugerir a importância do termo MA de quarto grau. Assim sendo, foram ajustados modelos $M A$ puros $\left(m_{5}\right.$ e $\left.m_{6}\right)$, os quais se mostraram superiores ao modelo misto $m_{3}$.

As funções $A C$ e $A C P$, para os dados de produção industrial, sugerem um componente $M A$ de primeira ordem e termos $A R$ de primeiro, segundo e terceiro graus. As estimativas efetuadas, bem como o teste RV, ambos resumidos na Tabela 6, mostram que o modelo misto $(3,1,1)$ é o que melhor reproduz o comportamento da série original.

Com respeito às taxas de juros, a função $A C$ apresenta um valor estatisticamente diferente de zero para uma defasagem de 5 periodos, o mesmo acontecendo com a função ACP nas defasagens 1 e 4 . Dentre os modelos estimados, cujos resultados são apresentados na Tabela $7, m_{3}$ e,$m_{4}$ mostraram-se os 
TABELA 2

LN DA PRODUÇÃO INDUSTRIAL, dados mensais, 1975-80: equações finais estimadas e testes da razäo de verossimilhança

\begin{tabular}{|c|c|c|c|c|c|c|c|c|c|c|c|}
\hline MODELOS & SQR & GL & $\mathrm{S} Q \mathrm{R} / \mathrm{GL}$ & $A R_{1}$ & $\mathrm{AR}_{2}$ & CONSTANTE & $\mathrm{MA}_{2}$ & $\bar{R}^{2}$ & $Q(12)$ & $Q(24)$ & $\mathbf{F}$ \\
\hline$m_{1}=(0,1,1) \times(0,1,0)_{12}$ & 0,07641 & 57 & 0,0013405 & & & $\begin{array}{r}-0,00091 \\
(-0,5574) \\
\end{array}$ & $\begin{array}{l}0,66911 \text { * } \\
(6,88)\end{array}$ & 0,348 & $\begin{array}{r}9,8 * k \\
(10 \mathrm{df})\end{array}$ & $\begin{array}{l}15,3 * \star \\
(22, \mathrm{df}) \\
\end{array}$ & $\begin{array}{l}F(1.57)= \\
a \quad 32 \star \star \star\end{array}$ \\
\hline$m_{2} \div(0,1,1) \times(0,1,0)_{12}$ & 0,07682 & 58 & 0,001324 & & & & $\begin{array}{l}0,66218 * \\
(6,77)\end{array}$ & 0,343 & $\begin{array}{r}9,8 * * \\
(11 d f) \\
\end{array}$ & $\begin{array}{l}15,2 \star \star \\
(23 \mathrm{df})\end{array}$ & $\begin{array}{l}F(1,58)= \\
=31,9 \star \star \star\end{array}$ \\
\hline$m_{3}:(2,1,0) \times(0,1,0)_{12}$ & 0,07509 & 57 & 0,001317 & $\begin{array}{l}-0,70772 \star \\
(-6,155)\end{array}$ & $\begin{array}{l}-0,41982 * \\
(-3,656)\end{array}$ & & & 0,358 & $\begin{array}{r}12,4 * * \\
(10 \mathrm{df}) \\
\end{array}$ & $\begin{array}{l}16,4 \star \star \\
(22 \mathrm{df})\end{array}$ & $\begin{array}{l}F(2,57)= \\
=17,5 * \star \star\end{array}$ \\
\hline $\mathrm{m}_{4}:(2,1,1) \times(0,1,0)_{12}$ & 0,07673 & 57 & 0,0013461 & & $\begin{array}{r}0,04359 \\
(-0,3137) \\
\end{array}$ & & $\begin{array}{l}0,64813 * \\
(5,93)\end{array}$ & 0,332 & $\begin{array}{r}9,6 * \star \\
(10 \mathrm{df}) \\
\end{array}$ & $\begin{array}{l}15,3 * \star \\
(22 \mathrm{df})\end{array}$ & $\begin{array}{l}\text { P }(2,57) \\
=15,7 * \star\end{array}$ \\
\hline $\mathrm{T}_{5}:(1,1,1) \times(0,1,0)_{12}$ & 0,07545 & 56 & 0,0013473 & $\begin{array}{r}-0,15917 \\
(-0,8747) \\
\end{array}$ & & & $\begin{array}{l}0,59446 * \\
(3,93)\end{array}$ & 0,349 & $\begin{array}{r}10,9 * * \\
(9 \mathrm{df})\end{array}$ & $\begin{array}{l}16 * * \\
(21 \mathrm{df})\end{array}$ & $\begin{array}{l}F(2,56)= \\
=16,5 \star \star \star\end{array}$ \\
\hline$m_{6}:(2,1,1) \times(0,1,0)_{12}$ & 0,07337 & 55 & 0,001334 & $\begin{array}{l}-0,41408 \\
(-1,599)\end{array}$ & $\begin{array}{l}-0,27407 \\
(-1,48)\end{array}$ & $\begin{array}{r}-0,00174 \\
(-0,5451) \\
\end{array}$ & $\begin{array}{c}0,35163 \\
(1,314) \\
\end{array}$ & 0,359 & $\begin{array}{l}12 \star \star \\
(8 \mathrm{df})\end{array}$ & $\begin{array}{l}17,2 \star \star \\
(20 \mathrm{df})\end{array}$ & $\begin{array}{l}F(3,55) \\
=11,8 \star \star \star \star\end{array}$ \\
\hline NODELOS COMPARADOS & & & $\lambda=\frac{L\left(X X_{1}\right)}{L\left(X H_{0}\right)}$ & & . & $2 \ln \lambda$ & & ? & & & . \\
\hline$H_{0}: \mathrm{s}_{4}$ vs $H_{1}: \mathrm{m}_{6}$ & & & 4,31261 & & & $2,92^{+}$ & & 2 & & & \\
\hline $\mathrm{H}_{0}: \mathrm{m}_{2}$ vs $\mathrm{H}_{1}: \mathrm{m}_{5}$ & & & 1,91136 & & & 1,29563 & & 2 & & & \\
\hline
\end{tabular}




\section{TABELA 3}

TAXAS DE JUROS PARA 6 MESES (custo para o mutuärio), dados mensais,1975-80:equaçōes finais estimadas e testes da razäo de verossiailhança

\begin{tabular}{|c|c|c|c|c|c|c|c|c|c|c|c|c|c|c|}
\hline MODELOS & SQR & GL & $\mathrm{SQR} / \mathrm{GL}$ & $\mathbf{A R _ { 1 }}$ & $A R_{4}$ & CONSTANTE & $\mathbf{M} \Lambda_{1}$ & $\mathrm{MA}_{2}$ & $\mathrm{KA}_{3}$ & $\overline{\mathrm{R}}^{2}$ & $Q(12)$ & $Q(24)$ & $F$ & \\
\hline$m_{1}:(0,1,3)$ & 9,81562 & 67 & 0,1465 & & & $\begin{array}{l}0,5694 \star \\
(2,9)\end{array}$ & $\begin{array}{l}0,35289 \text { * } \\
(3,873)\end{array}$ & $\begin{array}{l}-0,52035 \star \\
(-7,028)\end{array}$ & $\begin{array}{l}0,77009 \star \\
(8,189)\end{array}$ & 0,424 & $\begin{array}{r}7,7 \star \star \\
(8 \mathrm{df}) \\
\end{array}$ & $\begin{array}{l}11,7 \star \star \\
(20 \mathrm{df})\end{array}$ & $\begin{array}{l}F(3,67) \\
=18,2 \star \star \star \star\end{array}$ & 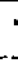 \\
\hline$\approx_{2}:(1,1,3)$ & 9,54227 & 66 & 0,14458 & $\begin{array}{l}-0,61254 \star \\
(-3,259)\end{array}$ & & $\begin{array}{l}0,09623 * \\
(2,173)\end{array}$ & $\begin{array}{r}-0,10627 \\
(-0,6119) \\
\end{array}$ & $\begin{array}{l}-0,24666 \star \\
(-2,041)\end{array}$ & $\begin{array}{l}0,43506 \star \\
(3,127)\end{array}$ & 0,4 & $\begin{array}{l}7,7 \star \star \\
(7 \mathrm{df})\end{array}$ & $\begin{array}{l}12,7 \star \star \\
(19 \mathrm{df})\end{array}$ & $\begin{array}{l}F(4,66) \\
=12,7 \star \star \star \star\end{array}$ & $=$ \\
\hline$m_{3}:(4,1,3)$ & 8,6398 & 65 & 0,13292 & $\begin{array}{r}0,04919 \\
(0,4152) \\
\end{array}$ & $\begin{array}{l}0,55284 \star \\
(4,603)\end{array}$ & $\begin{array}{l}0,02133 \star \\
(2,318)\end{array}$ & $\begin{array}{l}0,58153 \star \\
(13,79)\end{array}$ & $\begin{array}{l}-0,55741 \star \\
(-13,65) \\
\end{array}$ & $\begin{array}{c}0,90643 \star \\
(31,23)\end{array}$ & 0,489 & $\begin{array}{r}9,3 \star \star \\
(6 \mathrm{df}) \\
\end{array}$ & $\begin{array}{l}12,8 \star \star \\
(18 \mathrm{df})\end{array}$ & $\begin{array}{l}F(5,65) \\
-14,4 \star \star \star\end{array}$ & . \\
\hline $\mathbb{Q}_{4}:(4,1,3)$ & 8,6502 & 66 & 0,13166 & & $\begin{array}{l}0,54616 \star \\
(4,527)\end{array}$ & $\begin{array}{l}0,02405 * \\
(3,066)\end{array}$ & $\begin{array}{c}0,5564 \star \\
(12,3)\end{array}$ & $\begin{array}{l}-0,531527 \star \\
(-12,3)\end{array}$ & $\begin{array}{c}0,90565 * \\
(29,94)\end{array}$ & 0,491 & $\begin{array}{r}9,9 * \star \\
(7 \mathrm{df})\end{array}$ & $\begin{array}{l}13,5 * \star \\
(19 \mathrm{df})\end{array}$ & $\begin{array}{l}F(4,66) \\
=17,9 \star \star \star\end{array}$ & $=$ \\
\hline WDELOS COMPARADOS & & $\lambda=$ & $\frac{L\left(x H_{1}\right)}{L\left(X+H_{0}\right)}$ & & & $2 \ln \lambda$ & & & & $r$ & & & & \\
\hline$H_{0}: m_{1}$ vs $H_{1}: \square_{2}$ & & & 2,76425 & & & $2,03354^{+}$ & & & & 1 & & & & \\
\hline$H_{0}: w_{2}$ vs $H_{2}: D_{3}$ & & & 98,83385 & & & $9,18688^{++}$ & & & & 2 & & & & \\
\hline $\mathrm{H}_{0}: \mathrm{m}_{2}$ vs $\mathrm{H}_{3}: \mathrm{m}_{3}$ & & & $35+, 75431$ & & & $7,15334^{77}$ & & & & 1 & & & & \\
\hline $\mathrm{H}_{0}: \mathrm{m}_{1}$ vs $\mathrm{H}_{1}: \mathrm{m}_{4}$ & & & 94,64489 & & & $9,10026^{+1}$ & & & & 1 & & & & \\
\hline$H_{0}: m_{4}$ vs $H_{1}: m_{3}$ & & & 1,04426 & & & 0,08662 & & & & 1 & & & & \\
\hline
\end{tabular}


:AB!L $2: 4$

LN DA BASE MONET̃́RIA, dados mensais, 1975-80:equações

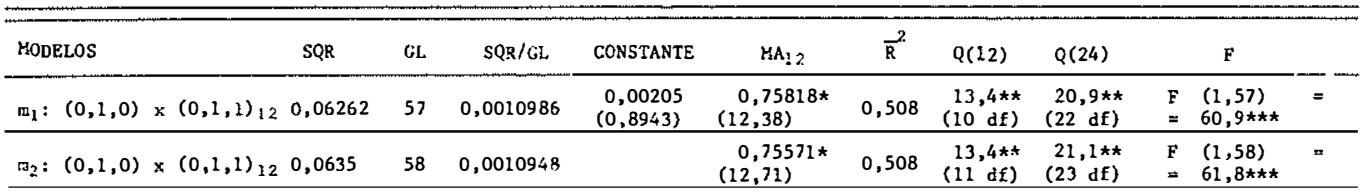


TABELA 5

LN DO NIVEL DE PREÇOS, dados trimestrais,1971-80:equaçöes finais estimadas e testés

\begin{tabular}{|c|c|c|c|c|c|c|c|c|c|c|c|c|c|}
\hline MODELOS & SQR & GL & $\mathrm{SQR} / \mathrm{GL}$ & $\mathrm{AR}_{4}$ & CONSTANTE & $\mathrm{MA}_{1}$ & $\mathrm{MA}_{4}$ & $\mathrm{MA}_{\mathrm{g}}$ & $\overline{\mathrm{R}}^{2}$ & $Q(12)$ & $Q(24)$ & $\mathbf{F}$ & \\
\hline$m_{1}:(4,2,8) \times(0,1,0)_{4}$ & 0,01834 & 32 & 0,0005731 & $\begin{array}{l}-0,7699 * \\
(-6,844)\end{array}$ & & & & $\begin{array}{l}0,79826 \star \\
(6,861)\end{array}$ & 0,456 & $\begin{array}{l}9,1 * \star \\
(10 \mathrm{df})\end{array}$ & $\begin{array}{l}16,2 \star \star \\
(22 \mathrm{df})\end{array}$ & $\begin{array}{l}F(2,32) \\
=15,3 \star \star \star\end{array}$ & $=$ \\
\hline$m_{2}:(4,2,1) \times(0,1,0)_{4}$ & 0.02387 & 32 & 0,0007459 & $\begin{array}{l}-0,49003 \star \\
(-3,101)\end{array}$ & & $\begin{array}{l}0,36805 * \\
(2,264)\end{array}$ & & & 0,234 & $\begin{array}{l}9,5 \star \star \\
(10 \mathrm{df})\end{array}$ & $\begin{array}{l}15,2 \star \star \\
(22 \mathrm{df})\end{array}$ & $\begin{array}{l}F(2,32) \\
=6,23 \star \star \star\end{array}$ & \\
\hline$m_{3}:(4,2,4) \times(0,1,0)_{z_{4}}$ & 0,01847 & 31 & 0,00059581 & $\begin{array}{r}-0,09477 \\
(-0,5052) \\
\end{array}$ & & $\begin{array}{l}-0,05781 \\
(-0,599) \\
\end{array}$ & $\begin{array}{l}0,8817 \star \\
(24,05)\end{array}$ & & 0,42 & $\begin{array}{r}11,9 \star \star \star \\
(9 \mathrm{df})\end{array}$ & $\begin{array}{l}18,9 \star \star \\
(21 \mathrm{df})\end{array}$ & $\begin{array}{l}F(3,31) \\
-\quad 9,26 \star \star \star\end{array}$ & $=$ \\
\hline$m_{4}=(4,2,8) \times(0,1,0)_{4}$ & 0,01829 & 31 & 0,00059 & $\begin{array}{l}-0,75404 \star \\
(-6,39)\end{array}$ & & $\begin{array}{l}-0,04131 \\
(-0,3105) \\
\end{array}$ & & $\begin{array}{l}0,81192 \star \\
(6,892)\end{array}$ & 0,44 & $\begin{array}{l}9,7 * \star \\
(9 \mathrm{df})\end{array}$ & $\begin{array}{l}17,1 * \star \\
(21 \mathrm{df})\end{array}$ & $\begin{array}{l}F(3,31) \\
=9,96 \star \star \star\end{array}$ & $=$ \\
\hline$m_{5}:(0,2,0) \times(0,1,1)_{4}$ & 0,01807 & 32 & 0,00056469 & & $\begin{array}{l}0,00164 \\
(1,174)\end{array}$ & & $\begin{array}{l}0,87843 * \\
(14,45)\end{array}$ & & 0,458 & $\begin{array}{l}11,4 \star \star \\
(10 \mathrm{df})\end{array}$ & $\begin{array}{l}18,2 \star \star \\
(22 \mathrm{df})\end{array}$ & $\begin{array}{l}F(1,32) \\
=28,9 \star \star \star\end{array}$ & w \\
\hline$m_{6}:(0,2,0) \times(0,1,1)_{4}$ & 0,0188 & 33 & 0,0005697 & $\cdot$ & & & $\begin{array}{c}0,86419 \text { * } \\
(15,5)\end{array}$ & & 0,451 & $\begin{array}{l}10,3 * \star \\
(11 \mathrm{df})\end{array}$ & $\begin{array}{l}16,7 \star \star \\
(23 \mathrm{df})\end{array}$ & $\begin{array}{l}F(1,33) \\
-29 \star \star \star\end{array}$ & 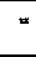 \\
\hline MODELOS COMPARADOS & & & \multicolumn{2}{|c|}{$\lambda=\frac{L\left(X \mathrm{H}_{1}\right)}{L\left(X \mathrm{H}_{0}\right)}$} & \multicolumn{3}{|c|}{$2 \ln \lambda$} & & $\tau$ & & & & \\
\hline$H_{0}: m_{2}$ vs $H_{1}: m_{3}$ & & & \multicolumn{2}{|c|}{168,93144} & \multicolumn{3}{|c|}{$10,25899+++$} & & 1 & & \multicolumn{3}{|c|}{$\cdot$} \\
\hline$H_{0}:$ 回l vs $H_{1}: \mathbb{R}_{4}$ & & & \multicolumn{2}{|c|}{1.056118} & \multicolumn{3}{|c|}{0,1092} & & 1 & & & & \\
\hline$H_{0}: m_{6}$ vs $H_{1}: m_{3}$ & & & \multicolumn{2}{|c|}{1,42501} & \multicolumn{3}{|c|}{0,70836} & & 2 & & & & \\
\hline
\end{tabular}


TABELA 6

LN DA PRODUÇ̃̃o INDUSTRIAL, dados

\begin{tabular}{|c|c|c|c|c|c|c|c|c|c|c|c|c|}
\hline MODELOS & $S Q R$ & GL & $\mathrm{SQR} / \mathrm{GL}$ & $\overline{\mathrm{AR}_{1}}$ & $\mathrm{AR}_{2}$ & $\mathrm{AR}_{3}$ & CONSTANTE & $M A_{1}$ & $\overline{\mathrm{R}}^{2}$ & $Q(12)$ & $Q(24)$ & $\mathbf{F}$ \\
\hline$m_{1}:(0,1,1)$ & 0,20934 & 37 & 0,0056578 & & & & $\begin{array}{l}0,02339 \star \\
(5,071)\end{array}$ & $\begin{array}{l}0,63497 * \\
(5,198)\end{array}$ & 0,29 & $\begin{array}{l}11,3 * * 2 \\
(10 \mathrm{df})\end{array}$ & $\begin{array}{l}14,6 \star t \\
(22 \mathrm{df})\end{array}$ & $\begin{array}{l}F(1,37) \\
=16,6 \star \star \star\end{array}$ \\
\hline$m_{2}:(3,1,0)$ & 0,18168 & 35 & 0,0051908 & $\begin{array}{l}-0,75946 \star \\
(-5,064) \\
\end{array}$ & $\begin{array}{l}-0,65646 \star \\
(-4,079) \\
\end{array}$ & $\begin{array}{l}-0,40708 \star \\
(-3,121)\end{array}$ & $\begin{array}{l}0,06768 \star \\
(4,585) \\
\end{array}$ & & 0,367 & $\begin{array}{c}4,4 \star \star \\
(18 \mathrm{df})\end{array}$ & $\begin{array}{l}7 * \star \\
(20 \mathrm{df})\end{array}$ & $\begin{array}{l}F(3,35) \\
-8,35 \star \star \star\end{array}$ \\
\hline$m_{3}:(3,1,1)$ & 0,16992 & 34 & 0,0049976 & $\begin{array}{l}-1,36473 \star \\
(-24,85) \\
\end{array}$ & $\begin{array}{l}-1,12553 \star \\
(-19,2)\end{array}$ & $\begin{array}{r}-0,7245 \\
(-8,354) \\
\end{array}$ & $\begin{array}{l}0,10224 \star \\
(5,323)\end{array}$ & $\begin{array}{l}-0,64242 \star \\
(-5,003)\end{array}$ & 0,416 & $\begin{array}{l}5,4 \star \star \\
(7 \mathrm{df}) \\
\end{array}$ & $\begin{array}{c}8,5 \star \star \\
(19 \mathrm{df})\end{array}$ & $\begin{array}{l}F(14,34) \\
=7,76 \star \star \star\end{array}$ \\
\hline MODELOS COMPARADOS & & & $=\frac{L\left(X H_{1}\right)}{L\left(X H_{0}\right)}$ & & & $2 \ln$ & & & $\mathbf{r}$ & & & \\
\hline$H_{0}: m_{1}$ vs $H_{1}: m_{3}$ & & & 64,88651 & & & 8,345 & $8+++$ & & 3 & & & \\
\hline$H_{0}: \mathrm{m}_{2}$ vs $\mathrm{H}_{1}: \mathrm{m}_{3}$ & & & 3,81287 & & & 2,676 & & & 1 & & & \\
\hline
\end{tabular}


TABELA 7

TAXAS DE JUROS ANUAIS (custo para o mutuärio), dados trimestrais,1971-80:equações finais estimadas e testes da razāo de verossimilhança

\begin{tabular}{|c|c|c|c|c|c|c|c|c|c|c|c|c|c|}
\hline MODELOS & SQR & G. & $\mathrm{SQR} / \mathrm{GL}$ & $\mathrm{AR}_{1}$ & ARr & CONSTANTE & $\mathrm{MA}_{1}$ & $\mathrm{MA}_{4}$ & $\mathbf{M A}_{5}$ & $\overline{\mathrm{R}}^{2}$ & $Q(12)$ & $Q(24)$ & $\mathbf{F}$ \\
\hline$\Phi_{1}:(4,1,1) \times(0,1,1)_{4}$ & 20,93512 & 30 & 0,697837 & $\begin{array}{l}0,558574 \star \\
(2,311)\end{array}$ & $\begin{array}{l}-0,49698 \star \\
(-2,248)\end{array}$ & $\begin{array}{l}0,09948 \\
(1,333) \\
\end{array}$ & $\begin{array}{c}0,23973 \\
(0,8258) \\
\end{array}$ & $\begin{array}{l}0,47929 \\
(1,666) \\
\end{array}$ & & 0,529 & $\begin{array}{r}5,5 * * \\
(7 \mathrm{df}) \\
\end{array}$ & $\begin{array}{l}10,1 * t \\
(19 \mathrm{df}) \\
\end{array}$ & $\begin{array}{l}F(4,30) \\
=10,6 * \star *\end{array}$ \\
\hline$\varpi_{2}:(0,1,5)$ & 21,4661 & 37 & 0,5801786 & & & $\begin{array}{l}0,27046 \star \\
(5,61.2)\end{array}$ & & & $\begin{array}{l}0,81733 * \\
(9,221)\end{array}$ & 0,377 & $\begin{array}{c}6,1^{\star \star} \\
\left(10^{\mathrm{df}}\right)\end{array}$ & $\begin{array}{r}10,9 \star * \\
(22 \mathrm{df})\end{array}$ & $\begin{array}{l}F(1,37) \\
=24 \star \star \star\end{array}$ \\
\hline $\mathbb{m}_{3}:(4,1,5) \times(0,1,0)_{4}$ & 18,70447 & 31 & 0,60337 & $\begin{array}{l}0,22761 \\
(1,355)\end{array}$ & $\begin{array}{l}-0,64685 \star \\
(-4,017)\end{array}$ & $\begin{array}{c}0,13179 \\
(1,716)\end{array}$ & & & $\begin{array}{l}0,59725 \star \\
(3,361)\end{array}$ & 0,594 & $\begin{array}{c}6,5 \star \star \\
(8 \mathrm{df}) \\
\end{array}$ & $\begin{array}{r}9,4 * * \\
(20 \mathrm{df}) \\
\end{array}$ & $\begin{array}{l}F(3,31) \\
=\quad 17,6 \star \star \star\end{array}$ \\
\hline $0_{4}:(4,1,5) \times(0,1,0)_{4}$ & 19,3399 & 32 & 0,6043719 & & $\begin{array}{l}-0,70785 \star \\
(4,528)\end{array}$ & $\begin{array}{l}0,15221 \star \\
(2,397)\end{array}$ & & & $\begin{array}{l}0,77584 \star \\
(7,802) \\
\end{array}$ & 0,598 & $\begin{array}{c}7,6 \star * \\
(9 \mathrm{df})\end{array}$ & $\begin{array}{r}11,9 \star * * \\
(21 \mathrm{df}) \\
\end{array}$ & $\begin{array}{l}F(2,32) \\
+\quad 26,2^{\star \star \star}\end{array}$ \\
\hline MODELOS COAPARADOS & & & $=\frac{L\left(X H_{1}\right)}{L\left(X H_{0}\right)}$ & & & $2 \ln \lambda$ & & & & $\mathbf{r}$ & & & \\
\hline $\mathrm{H}_{0}: \mathrm{O}_{4}$ vs $\mathrm{H}_{1}: \mathrm{m}_{3}$ & & & 1,95064 & & & $1,33631 *$ & & & & 1 & & & \\
\hline
\end{tabular}


mais adequados, com o teste RV rejeitando $m_{3}$ a um nivel de significância de $25 \%$. Uma opção clara por $m_{3}$ ou $m_{4}$ não é de muita relevância, pois o que diferencia os dois modelos é o termo $A R$ de primeiro grau e não a ordem do polinômio autoregressivo; em ambos os casos o processo escolhido é $(4,1,5) \times$ $(0,1,0)_{4}$

Os resultados obtidos para a série de base monetária encontram-se na Tabela 8. Os valores das funções $A C$ e $A C P$ sugerem um componente sazonal e termos $A R$ ou $M A$ de primeiro grau. $O$ teste $R V$ indica que o modelo $(1,1,1) X$ $(0,1,1)_{4}$ possui um número excessivo de parâmetros, optando-se portanto por $m_{1}$ e $m_{3}$, com alguma preferência pelo processo $M A$

No que tange às funções de transferência, o exame das correlações cruzadas não indicou a existência de relações sistemáticas, em temos mensais, entre as variáveis endógenas e a base monetária. De acordo com Box e Jenkins, as séries relativas às variáveis endógenas foram filtradas através do mesmo processo ARMA ajustado à série da variável exógena (base monetária), examinando-se em seguida as correlações cruzadas entre os resíduos. Os poucos valores estatisticamente diferen. tes de zero apresentaram padrões bastante irregulares, não indicando a possibilidade de ajustamento de fiunções de transferência. Este resultado não chega a ser surpreendente; não seria mesmo razoável esperar que flutuações mensais na taxa de expansão da base monetária produzissem efeitos sistemáticos sobre o produto real, a taxa de juros e a taxa de inflação. Com dados trimestrais as relações tornaram-se mais claras, mas não a ponto de permitir modelagem.

Em termos semestrais, considerando-se o semestre que termina a cada mês, ( 4 ) os resultados são bem melhores. Optou-se por esta agregação, e não por dados referentes a semestres legais, com o intuito de preservar um número aceitável de graus de liberdade. Para evitar a falsa causalidade, foram examinados apenas os valores das funções de correlação cruzada para as defasagens $6 k, k=1,2, \ldots$ As funções estimadas foram:

$$
\begin{aligned}
& \begin{aligned}
\left(1-L^{6}\right) r p_{t}= & \left(0,14+0,17 L^{6}\right)\left(1-0,39 L^{12}\right)\left(1-L^{6}\right) r h_{t}+0,02+e_{1 t} \\
& (1,718) \quad(2,06)
\end{aligned}
\end{aligned}
$$

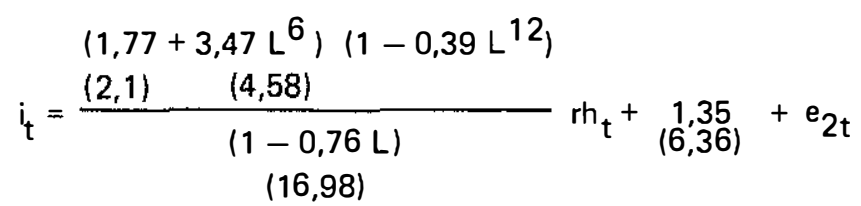

(4)

A observação para o mês é o ln da média da séric original nos últimos seis meses. 
TABELA 8

LN DA BASE MOETTRRIA,dados trimestrais,1971-80:equações finais estinadas e testes da razão de verossinilhança

\begin{tabular}{|c|c|c|c|c|c|c|c|c|c|c|c|}
\hline MODELOS & $S Q R$ & GL & $S Q R / G L$ & $\mathbf{A R}_{\mathbf{1}}$ & CONSTANTE & $M A_{1}$ & $\mathbf{M A}_{\mathbf{L}_{4}}$ & $\overrightarrow{R^{2}}$ & $Q(12)$ & $Q(24)$ & $\mathbf{F}$ \\
\hline$m_{1}:(1,1,0) \times(0,1,1)_{4}$ & 0,05285 & 33 & 0,0016019 & $\begin{array}{l}0,43982 \star \\
(2,757)\end{array}$ & & & $\begin{array}{l}0,80078 * \\
(11,79)\end{array}$ & 0,471 & $\begin{array}{l}4,7 \star \star \\
(10 \mathrm{df})\end{array}$ & $\begin{array}{l}12,2 \star \star \\
(22 \mathrm{df})\end{array}$ & $\begin{array}{l}F(2,33) \\
=16,6 \star \star \star\end{array}$ \\
\hline D $_{2} \cdot(0,1,1) \times(0,1,1)_{z_{1}}$ & 0,04757 & 32 & 0,00148656 & & $\begin{array}{l}0,00598 \\
(1,852)\end{array}$ & $\begin{array}{l}-0,39667 \star \\
(-2,313)\end{array}$ & $\begin{array}{l}0,81624 \\
(12,55)\end{array}$ & 0,531 & $\begin{array}{l}5,4 \star \star \\
(9 \mathrm{df})\end{array}$ & $\begin{array}{l}12,8^{\star \star \star} \\
\left(2 I^{d f}\right)\end{array}$ & $\begin{array}{l}F(2,32) \\
=20,2 \star \star \star\end{array}$ \\
\hline$m_{3}:(0,1,1) \times(0,1,1)_{4}$ & 0,05186 & 33 & 0,0015715 & & & $\begin{array}{l}-0,50246 * \\
(-3,267)\end{array}$ & $\begin{array}{l}0,76549 * \\
(9,795)\end{array}$ & 0,484 & $\begin{array}{l}3,6 * \star \\
(10 \mathrm{dE})\end{array}$ & $\begin{array}{l}10,2 \star \star \\
(22 \mathrm{df})\end{array}$ & $\begin{array}{l}\text { F }(2,33) \\
=17,5 * k *\end{array}$ \\
\hline$D_{4}:(1,1,3) \times(0,1,1)_{4}$ & 0,05181 & 32 & 0,0016191 & $\begin{array}{c}0,10198 \\
(0,2785) \\
\end{array}$ & & $\begin{array}{r}-0,41001 \\
(-1,228) \\
\end{array}$ & $\begin{array}{l}0,78289 * \\
(9,893)\end{array}$ & 0,468 & $\begin{array}{l}3,6 * \star \\
(9 \mathrm{~d} f)\end{array}$ & $\begin{array}{l}10,4 * * \\
(21 d f)\end{array}$ & $\begin{array}{l}F(3,32) \\
=11,3 * \pi *\end{array}$ \\
\hline MODELOS COAPARADOS & & $\lambda=$ & $\frac{L\left(x H_{1}\right)}{L\left(X H_{0}\right)}$ & & & $2 \ln \lambda$ & & $\mathbf{r}$ & & & \\
\hline $\mathrm{H}_{0}: \mathrm{m}_{1}$ vs $\mathrm{H}_{1}: \mathrm{rl}_{4}$ & & & 1,48809 & & & 0,79498 & & 1 & & & \\
\hline$H_{0}: \Xi_{3}$ vs $H_{1}: \Xi_{4}$ & & & 1,01948 & & & 0,03858 & & 1 & & & \\
\hline
\end{tabular}


Um resultado bastante interessante e que pode ser entendido a partir do conceito de expectativas racionais, segundo o qual apenas variações não-antecipadas na oferta de moeda afetam variáveis reais, é a ausência de uma relação sistemática entre a taxa de expansão da base monetária e a taxa de crescimento da produção industrial. Um exame das correlações cruzadas entre estas variáveis, uma vez filtradas pelo processo estocástico estimado para a base monetária, não sugere o ajustamento de uma função de transferência.

Os modelos ARMA sugeridos pela análise empirica encontram-se na

Tabela 9 .

\section{CONSIDERAÇÕES FINAIS}

A comparação entre as ordens esperadas a partir dos modelos monetários examinados na seção 3 e aquelas verificadas empiricamente, leva a algumas observações:

(i) em termos mensais, as ordens esperadas para os polinômios AR e MA são sistematicamente maiores do que as ordens encontradas na análise empirica, independentemente do mecanismo de formação de expectativas considerado;

(ii) as ordens esperadas para os polinômios AR nos modelos de expectativas racionais, são extremamente compatíveis com os resultados da análise dos dados trimestrais; a implicação, aparentemente restritiva, de que, salvo nos casos de cancelamento de termos comuns, os polinômios AR nas equações finais das variáveis endógenas são idênticos, é parcialmente satisfeita para inflação $\left(m_{1}\right.$ na Tabela 5 ) e taxa de juros $\left(m_{4}\right.$ na Tabela 7$)$;

(iii) as ordens esperadas para os polinômios MA de todos os modelos são sempre maiores do que aquelas indicadas pelos resultados empíricos, o que sugere estruturas teóricas de erros menos complexas. Estas estruturas teriam sido obtidas caso tivesse sido adotado neste trabalho o procedimento de Zellner e Palm (1975), de introduzir as perturbações aleatórias nas equações estruturais somente após a eliminação da variável não-observável;

(iv) exceto no caso do modelo com expectativas adaptativas em que não se considera a persistência no hiato do produto real, as ordens esperadas para os polinômios AR e MA nas equações finais da taxa de juros correspondem exatamente àquelas encontradas na análise dos dados trimestrais;

(v) o modelo generalizado com expectativas racionais integra a prática econométrica usual à análise de séries temporais: a teoria econômica é utilizada na especificação da forma estrutural, cabendo à análise dos dados a determinação da estrutura de defasagens dos polinómios de ajustamento. Caso a evidência empírica houvesse mostrado, que o conjunto das variáveis endógenas do modelo tem seu comportamento ao longo dos ciclos econômicos deterninado por um polinômio $A R$ de grau superior a 4 , os dados teriam realmente transmitido alguma informa- 
ção a respeito do "polinômio de persistência" $\psi_{1}(L)$. No entanto, a dinâmica introduzida na estrutura do modelo mais simples com expectativas racionais revelou-se suficientemente complexa para reproduzir as informações contidas nos dados.

\section{REFERÊNCIAS BIBLIOGRÁFICAS}

Box, G.E.P. and Jenkins, G.M. - Time Series Analysis Forecasting and Control, 1970, Holden-Day Inc., San Francisco.

Lucas, R. - "Some International Evidence on Output-Inflation Tradeoffs", American Economic Review, 1973, 68, 326-334.

Quenouille, M.H. - The Analysis of Multiple Time Series, 1957, C. Griffin and Co., London.

Sargent, T. - "A Classical Macroeconomic Model of the United States", Journal of Political Economy, 1976, 84, 207-238.

Theil, H. and Boot, J.C.D. - "The Final Form of Econometric Equation Systems", Review of the International Statistical Institute, 1962, 30, 136- 152.

Tinbergen, J. - "Econometric business cycle research", Review of Economic Studies, 1940, 7, 73-90.

Zellner, A. and Palm, F. - "Time Series Analysis and Simultaneous Equation Models", Journal of Econometrics, 1974, 2, 17-54.

Zellner, A. and Palm, F. - "Time Series and Structural Analysis of Monetary Models of the U.S. Economy", Sankyá (The Indian Journal of Statistics), 1975, 37. Series C, Pt. 2, 12-56 